\title{
The in-flight monitoring and validation of the SOHO CDS Normal Incidence Spectrometer radiometric calibration
}

\author{
J. Lang ${ }^{1}$, D. H. Brooks ${ }^{2, \star}$, A. C. Lanzafame ${ }^{3}$, R. Martin ${ }^{4}$, C. D. Pike ${ }^{1}$, and W. T. Thompson ${ }^{5}$ \\ ${ }^{1}$ CCLRC Rutherford Appleton Laboratory, Chilton, Didcot, Oxon. OX11 0QX, UK \\ e-mail: J.Lang@rl.ac.uk \\ 2 E. O. Hulburt Center for Space Research, Code 7670, Naval Research Laboratory, Washington, DC 20375, USA \\ Dipartimento di Fisica e Astronomia, Università di Catania, via S. Sofia 78, 95123 Catania, Italy \\ 4 Department of Physics, University of Strathclyde, 107 Rottenrow, Glasgow G4 0NG, UK \\ 5 L-3 Communications GSI, NASA GSFC, Code 612.1, Greenbelt, MD 20771, USA
}

Received 4 November 2005 / Accepted 14 November 2006

ABSTRACT

\begin{abstract}
The scientific return from an extreme-ultraviolet spectrometer depends on the accuracy and precision of its radiometric calibration. For the Coronal Diagnostic Spectrometer on SOHO, radiometric calibration started pre-launch in the laboratory and continued after launch by making comparison measurements of the same area of the Sun with calibrated sounding rocket payloads and also by intercalibration with the SUMER instrument on SOHO. The present work uses the measurement of line ratios to monitor and validate the calibration over the first six years of observation. As well as using branching ratios and line ratios independent of the electron temperature and density, line ratios dependent on electron temperature or density have also been used successfully to validate and monitor the calibration. The results indicate that, within the uncertainties, the radiometric calibration has been validated and maintained over the first six years of observations apart from three specific wavelengths, $338.98 \AA, 315.0 \AA$, and $311.8 \AA$. Problems with lines at $608.4 \AA$, $303.4 \AA$ (seen in second order), $335.4 \AA$, and $360.7 \AA$ are attributed to difficulties with the burn-in correction.
\end{abstract}

Key words. Sun: atmosphere - Sun: corona - Sun: UV radiation - atomic data - techniques: spectroscopic

\section{Introduction}

The radiometric calibration of the SOHO Coronal Diagnostic Spectrometer (CDS) Normal Incidence Spectrometer (NIS) is fundamental to the optimum exploitation of the observations. A well calibrated instrument with well understood and quantified uncertainties is necessary to derive plasma parameters such as the electron temperature and density, by the line ratios method, with the best accuracy and precision. CDS was calibrated in the laboratory before launch and since launch much effort has been expended in refining the calibration. In-flight studies have included inter-comparisons with the other instruments on SOHO and sounding rocket flights as well as studies based solely on NIS observations. The radiometric calibration of SOHO has been reported by Pauluhn et al. (2002a). The development and present status of the radiometric calibration of CDS is given by Lang et al. (2002).

There have been two studies devoted explicitly to investigating the calibration of the CDS NIS. Landi et al. (1997) used a spectroscopic method to determine discrepancies in the NIS calibration from observations taken in the first two months of operations. This work, together with preliminary results from the laboratory calibration, was the basis of version 1 of the NIS calibration. Del Zanna et al. (2001) used both CDS NIS and Grazing Incidence Spectrometer (GIS) observations to evaluate a relative radiometric calibration of both the NIS and GIS. Their observations were taken on ten occasions before the loss-of-attitude of $\mathrm{SOHO}$ and one following recovery of the

^ Also George Mason University, 4400 University Drive, Fairfax, VA 22030, USA. spacecraft. As pointed out in Lang et al. (2002) the calibration has been revised since either analysis was done and the detector burn-in corrections (see Sect. 4), particularly that for the wide slit, are now improved. Thus, in making comparisons with results from either paper, care must be exercised and indeed using their calibrations will give anomalous results. More recently, Lanzafame et al. (2005) made a study of the differential emission measure (DEM) of the quiet Sun using observations taken on 1996 December 5 and were able to place limits on the uncertainty of the NIS calibration.

This paper describes the in-flight monitoring and validation of the NIS radiometric calibration using NIS observations taken over a number of years. Any possible changes to the calibration arising during the four month period in 1998 when attitude control of SOHO was lost and had to be recovered are also investigated. During the loss and much of the recovery CDS was at a temperature of around $100{ }^{\circ} \mathrm{C}$, well above the $0{ }^{\circ} \mathrm{C}$ to $40{ }^{\circ} \mathrm{C}$ over which it had been tested prior to launch. This baking caused an irreversible distortion in the NIS, causing the line profiles to alter and the location of the spectra on the detector to change. In the next section a brief overview of CDS is given, then comes a section on the methods used. The following sections describe the observations and the data analysis. Subsequent sections deal with the calibration analysis and the last section contains a discussion of the results and the conclusions.

\section{Overview of CDS}

CDS has been fully described by Harrison et al. (1995) and only the details pertinent to this work are given. 
A Wolter-Schwarzschild grazing incidence telescope is used to focus the solar image, via a plane scan mirror, at the common entrance slit of a twin spectrometer. Two apertures before the telescope, and light stops after, define the areas of the telescope used and the illumination of the spectrometers through the slit assembly. In the NIS, light from the slit is incident on a pair of toroidal gratings at near normal incidence. The gratings are slightly tilted with respect to each other so that the two stigmatic diffracted images of the slit can be detected simultaneously on a single two-dimensional detector. The detector is a micro-channel plate intensified CCD and has been described by Thompson et al. (1992). The wavelength ranges of the two bands are $308 \AA$ to $381 \AA$ (hereafter referred to as NIS-1) and $513 \AA$ to $633 \AA$ (hereafter referred to as NIS-2). The spectrometer entrance slit is aligned in the solar N-S direction. Two dimensional images are obtained by moving the scan mirror in the W-E direction. The field of view of the instrument is $4^{\prime}$ by $4^{\prime}$ and can cover anywhere on the Sun by moving the instrument's articulated legs.

\section{Method}

Various spectroscopic analyses can be applied to obtain a relative radiometric calibration of CDS NIS. The best direct method, in terms of the precision achievable, is known as the branching ratios method (Griffin \& McWhirter 1962). This method allows an intensity calibration to be transferred from one spectral range where it is known to another where it is needed, by making measurements of suitable spectral line pairs. These line pairs must satisfy several criteria, namely, they must arise from common upper levels, have well calculated or measured branching ratios of spontaneous radiative transition probabilities (A values) and must lie one in each of the relevant spectral ranges. Neupert \& Kastner (1983) extended this to include line ratios which were insensitive to the plasma conditions, i.e., electron temperature and density. From the standpoint of minimising uncertainties, the branching ratios method is to be preferred since only A values from a common upper level are needed for any line pair and these are known to high precision. To derive the insensitive ratios, a model ion is required and thus a combination of electron excitation rate coefficients is needed as well as the A values. The uncertainty of the resulting ratio then includes uncertainties in both the collision rates and the A values and those for the former are larger than those for the latter. In the present application of the methods, since the calibration is already established, it is checked by comparing the measured line intensity ratios with their predictions.

Suitable line ratios are limited in number, so we also investigate the use of line ratios sensitive to the plasma electron density or electron temperature, or both.

The work of Landi et al. (1997) involved determining the DEM distribution and by comparing the intensities from NIS-1 and NIS-2 they were able to determine that the NIS-1 calibration gave persistently low intensities and needed updating. This approach is not used. Instead the results of Lanzafame et al. (2005) are used. They studied the DEM of quiet Sun regions, obtaining the DEMs from well observed lines with reliable atomic data, and forward predicted the intensities of other lines. The comparison was then made with not only predicted and observed intensities but also predicted and observed line ratios. Their work allowed limits to be placed on the NIS-1/NIS-2 cross calibration, and suggested that the uncertainty in the cross calibration is at most $10 \%$. Our quiet Sun line ratio results are compared to theirs.

\section{Observations and data analysis}

Data from the observing sequence NISAT_S (ID17 variation 2) were used for the present analysis. This sequence builds up a raster using the $2^{\prime \prime}$ by $240^{\prime \prime}$ slit by moving it to 10 adjacent locations to give an area on the Sun of $20^{\prime \prime}$ by $240^{\prime \prime}$ (ignoring contributions from solar rotation). The exposure time at each raster step was $50 \mathrm{~s}$ and, allowing for the time taken to transmit the data to the ground, the whole sequence took $60 \mathrm{~min}$ to complete. The full spectral ranges of both channels were observed and the use of the narrowest slit optimised the spectral resolution. The sequence is run at least every week on the quiet Sun and at least every month on both active regions and coronal holes. All observations from March 1996, the start of CDS operations, till May 2002 are included in our dataset. The area of the Sun observed was checked by over-plotting the raster area on images of the Sun obtained by the EUV Imaging Telescope (EIT) (Delaboudinière et al. 1995). Mostly the EIT Fe XII $195 \AA$ band was used. Full-Sun and magnified images were used and each of four equal sections along the length of the slit classified as quiet Sun, active region, coronal hole or above limb. The division was done to aid unique assessment since in many of the observations raster areas included, e.g., both coronal hole and quiet Sun regions.

The NIS data in each observation require debias correction, spectral rotation and line tilt correction, cosmic ray cleaning, flat fielding to allow for the pixel-to-pixel variation in detector responsivity (a few percent) and, in a few instances, corrections for non-linearity in detector response. As the detector micro-channel plate suffers from degradation when exposed to radiation, its responsivity declines with photons detected, i.e., effectively time. The NIS optical elements are fixed so the emission lines lie in more or less the same area and the degradation, i.e., burn-in, is greatest for the strongest lines. The widths of some lines are also affected and for this a correction (tied to the burn-in correction) is available. The change of location of the spectra on the micro-channel plate because of the elevated temperatures during the loss of SOHO's attitude control also meant that a new postrecovery burn-in correction had to be derived. The radiometric calibration as detailed in Lang et al. (2002) was also applied.

For each observation, the corrected and calibrated data were averaged over the 10 mirror positions in the raster. To avoid any possible edge effects the lowest 15 and highest 9 pixels were ignored and the remaining 120 divided to give four equal segments each of 50.4" along the length of the slit. The data were stored in four arrays to allow discrimination between NIS-1 and NIS-2 and before and after the SOHO loss-of-attitude. The spectral line fitting was done using the program ADAS602 in the Atomic Data and Analysis Structure (ADAS) (Summers 1994, 2001). In the program (Lang et al. 1990; Brooks et al. 1999) the relevant portions of the observed spectrum can be fitted with a background and up to ten lines. For observations taken before the loss-of-attitude the profiles are Gaussian while for observations after the loss-of-attitude, the profiles are modified Gaussians appropriate for NIS-1 or NIS-2. The parameters of the fit, including estimates of their standard errors, are obtained by the maximum likelihood method.

Since only line intensity ratios are used for the NIS, the uncertainty budget for each line is the burn-in uncertainty of $10 \%$ with an additional $10 \%$ for observations taken after SOHO's loss-of-attitude control as well as the uncertainty from the line fitting program. Also needed are the uncertainties for the wavelength dependent count/photon conversion as given by Lang et al. (2002). For NIS-1 these relative uncertainties are $15 \%$ 
below $368 \AA$ and $25 \%$ above $368 \AA$. For NIS-2 they are $18 \%$ at $584 \AA$ and $29 \%$ at the ends of the bands. Linear interpolation was used between $584 \AA$ and the ends of the band. For NIS- 2 in second order the uncertainties are $12 \%$ at $303 \AA$ to $304 \AA$. These suffice for ratios within each band but for ratios between NIS-1 and NIS-2 lines, the uncertainties in the NIS-1 and NIS-2 aperture measurements must also be added. These are each $8 \%$ as given in Lang et al. (2000).

\section{Monitoring the calibration of NIS-2}

\subsection{OIII}

The O III lines at $525.8 \AA\left(2 \mathrm{~s}^{2} 2 \mathrm{p}^{2}{ }^{1} \mathrm{D}_{2}-2 \mathrm{~s} 2 \mathrm{p}^{3}{ }^{1} \mathrm{P}_{1}\right)$ and $597.8 \AA$ $\left(2 \mathrm{~s}^{2} 2 \mathrm{p}^{2}{ }^{1} \mathrm{~S}_{0}-2 \mathrm{~s} 2 \mathrm{p}^{3}{ }^{1} \mathrm{P}_{1}\right)$ form a branching ratio and have a good wavelength span, covering $72 \AA$ of the NIS- 2 wavelength range of $120 \AA$. Landi et al. (1997) and Del Zanna et al. (2001) used the $525.8 \AA$ line with the $599.6 \AA$ line (the $2 \mathrm{~s}^{2} 2 \mathrm{p}^{2}{ }^{1} \mathrm{D}_{2}-2 \mathrm{~s} 2 \mathrm{p}^{3}{ }^{1} \mathrm{D}_{2}$ transition) although the latter noted that the weak $597.8 \AA$ line was "always in good agreement with the other two lines". For the theoretical ratios the A values are taken from the critical compilation by Weise et al. (1996) who rate the uncertainties of the recommended values as $\mathrm{A}$ and $\mathrm{A}-$ - In this compilation $\mathrm{AA}, \mathrm{A}$ and $\mathrm{B}$ represent uncertainties within $1 \%, 3 \%$ and $10 \%$ respectively. A- is interpreted as $5 \%$, yielding $6 \%$ for the uncertainty in the ratio. This could be a conservative estimate as the transitions emanate from a common upper level and problems with any calculations should tend to cancel.

One small problem with the ratio is that the $597.8 \AA$ transition is blended with the weak $3 / 2-3 / 2$ component of the $3 s^{2} 3 p{ }^{2} \mathrm{P}_{\mathrm{J}}-3 \mathrm{~s} 3 \mathrm{p}^{2}{ }^{2} \mathrm{D}_{\mathrm{J}}$ multiplet of Ca VIII at $597.85 \AA$. The $1 / 2-3 / 2$ component of the Ca VIII multiplet at $582.84 \AA$ forms a branching ratio with the blended component but lies in the wings of the strong He II line at $584.3 \AA$ and the calibration changes over the wavelength range between the lines. The $3 / 2-5 / 2$ component of the Ca VIII multiplet is at $596.94 \AA$ and the calibration changes by about $0.4 \%$ between this line and the Ca VIII/O III blend. Landi et al. (2004) give the correction required. However, it is not consistent with the branching ratio as given in their table of A values. This discrepancy is probably because the intensity of the blended line was recovered from the DEM rather than the unblended branching line using the A value ratio. The correction factor, that is the factor by which the intensity of the $3 / 2-5 / 2$ component should be divided to give the Ca VIII contribution to its blend with the O III line, was evaluated as a function of density for three temperatures using the ADAS codes ADAS205 and ADAS207. The temperatures used were the temperature of maximum ion population in ionisation equilibrium for Ca VIII (as given by Arnaud \& Rothenflug (1985)), the temperature two times larger and the temperature two times smaller. The correction factor at the maximum ion population in ionisation equilibrium $\left(5.6 \times 10^{5} \mathrm{~K}\right)$ is 14.0 for densities greater than $1 \times 10^{9} \mathrm{~cm}^{-3}$, i.e., for active regions. However, at lower densities, i.e., for the quiet Sun, the correction factor is density sensitive, decreasing with decreasing density. It is $12.5,7.2$ and 5.5 at densities of $1 \times 10^{8} \mathrm{~cm}^{-3}$, $1 \times 10^{7} \mathrm{~cm}^{-3}$ and $5 \times 10^{6} \mathrm{~cm}^{-3}$ respectively. There is very little temperature sensitivity of the correction factor above densities of $1 \times 10^{9} \mathrm{~cm}^{-3}$, a factor two change in temperature from the ionisation balance temperature giving a $4 \%$ change. If the upper levels of the multiplet were statistically populated and followed the tabulations of Allen (1976), based on the assumptions of

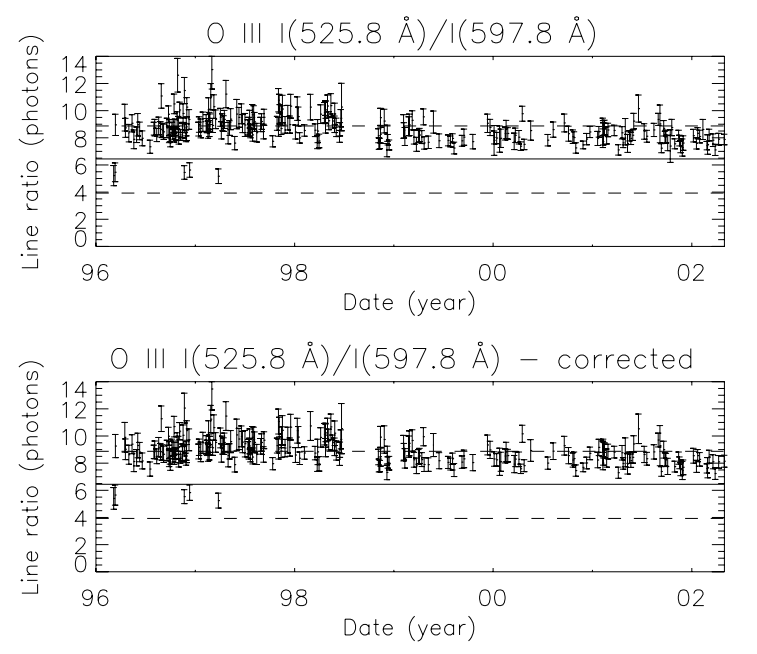

Fig. 1. The O III line intensity ratio $I(525.8 \AA) / I(597.8 \AA)$. In each graph, the solid line parallel to the abscissa is the theoretical branching ratio while the dashed lines represent the upper and lower ranges as determined by the addition in quadrature of the uncertainties in the branching ratio and for each line the burn-in correction and the radiometric calibration. The uncertainties in the measured ratios are obtained from the uncertainties as determined by the fitting routine, including those of the correction line and the correction factor in the blend corrected ratio.

Russell-Saunders coupling, the correction factor would be nine. This is the value adopted here.

Figure 1 shows the ratio and the blend corrected ratio over the first six years of observation. The gap in 1998 when the spacecraft's attitude control was lost can easily be identified. As can be seen from Fig. 1, the blend correction is small, the corrected and uncorrected averages being 3\% different. Taking the correction factor to have an uncertainty of $50 \%$ makes a percent or two difference to the uncertainties of the measured ratios.

The line intensity ratio $I(525.8 \AA) / I(597.8 \AA)$ was also measured for active regions, i.e., where the correction factor is independent of electron density. However, the weighted averages of the corrected active region ratios are $10 \%$ greater than those for the quiet Sun. This indicates a possible blending of the $525.8 \AA$ line in active regions. The blend may also be present for some of the quiet Sun ratios causing the ratio to be high, as is observed. This blend would also affect the $525.8 \AA$ to $599.6 \AA$ intensity ratio, as used by Landi et al. (1997) and Del Zanna et al. (2001), which is a density insensitive ratio in active regions. This ratio also has higher theoretical uncertainties than the branching ratio used here. If a simple atomic model is used whereby the upper levels of the transitions are only excited from the $2 \mathrm{~s}^{2} 2 \mathrm{p}^{2}{ }^{1} \mathrm{D}_{2}$ metastable level, with uncertainties of around $15 \%$ for the excitation rate coefficients with no contributions from the ground term, the ratio has uncertainties of about $20 \% \mathrm{cf}$. $6 \%$ for the branching ratio used here. This is thus much less sensitive to the calibration.

Our uncorrected results agree with those of Lanzafame et al. (2005). The agreement between the measured ratios and the theory is reasonable, especially noting the possible blend of the $525.8 \AA$ line. Moreover, this agreement is maintained for six years and is only slightly changed before and after the lossof-attitude of the spacecraft in 1998. 


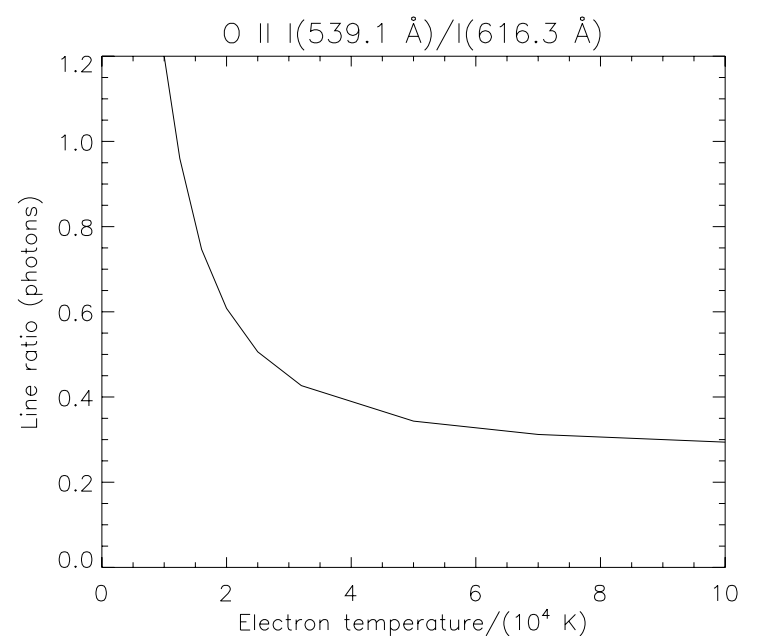

Fig. 2. The O II line intensity ratio $I(539.1 \AA) / I(616.3 \AA)$ as a function of electron temperature for an electron density of $1 \times 10^{8} \mathrm{~cm}^{-3}$.

\section{2. $0 ॥$}

The ratio of the intensity of the $3 / 2-5 / 2$ or the blend of the $3 / 2-3 / 2$ and $3 / 2-1 / 2$ components of the O II $2 s^{2} 2 p^{3}{ }^{4} \mathrm{~S}-2 \mathrm{~s}^{2} 2 \mathrm{p}^{2} 3 \mathrm{~s}{ }^{4} \mathrm{P}$ multiplet at $539.1 \AA$ or $539.6 \AA$ to the intensity of the blend of the 5/2-3/2 and 3/2-3/2 components of the O II $2 \mathrm{~s}^{2} 2 \mathrm{p}^{3}{ }^{2} \mathrm{D}-2 \mathrm{~s}^{2} 2 \mathrm{p}^{2} 3 \mathrm{~s}{ }^{2} \mathrm{P}$ multiplet at $616.3 \AA$ gives a good wavelength spread, $77 \AA$. This ratio has not been considered before for calibration purposes as it is temperature sensitive. The theoretical line ratio $I(539.1 \AA) / I(616.3 \AA)$ is shown in Fig. 2 as a function of temperature for a density of $1 \times 10^{8} \mathrm{~cm}^{-3}$. The temperature of maximum O II population in the ionisation equilibrium calculations of Arnaud \& Rothenflug (1985) is $3.2 \times 10^{4} \mathrm{~K}$. This distribution falls to zero by $1.0 \times$ $10^{4} \mathrm{~K}$ and $1.6 \times 10^{5} \mathrm{~K}$. In the atomic model used in ADAS to obtain the line ratios, the majority of the A values were taken from Bell et al. (1994). The effective collision strengths involving transitions within the $2 \mathrm{p}^{3}$ configuration were taken from McLaughlin \& Bell (1998) and the others from McLaughlin \& Bell (1994). The calculations were done in LS coupling and the line intensities derived from the multiplet values assuming the upper levels of the transitions were statistically populated.

Figure 3 shows O II line intensity ratios. The ratio $I(539.1 \AA) / I(539.6 \AA)$ is one. The uncertainty in the ratio is taken as $20 \%$, including an allowance for the assumption that the upper levels of the transitions are statistically populated. Only the burn-in correction uncertainties are added to these as the lines are very close in wavelength and the radiometric calibration is effectively the same for each line. The ratios before and after the loss of spacecraft attitude are constant but different, the before-loss ratios agreeing better with theory. Comparison with the other two ratios indicates that the change can be ascribed to an increase in intensity of the $539.1 \AA$ line by around $15 \%$.

The measured temperature sensitive ratio $I(539.6 \AA) /$ $I(616.3 \AA)$ shows little scatter. In this case the A values and effective collision strengths used were considered to have uncertainties of $10 \%$ and $30 \%$ respectively. The difference between the measured ratios and that expected at a density of $1 \times$ $10^{8} \mathrm{~cm}^{-3}$ and a temperature of $3.2 \times 10^{4} \mathrm{~K}$, the temperature of maximum ion population in ionisation equilibrium, is attributed to the atomic data and most likely due to the $\Delta n=1$ effective collision strengths and the assumption that the upper levels of the transitions are statistically populated. There is a slight fall in

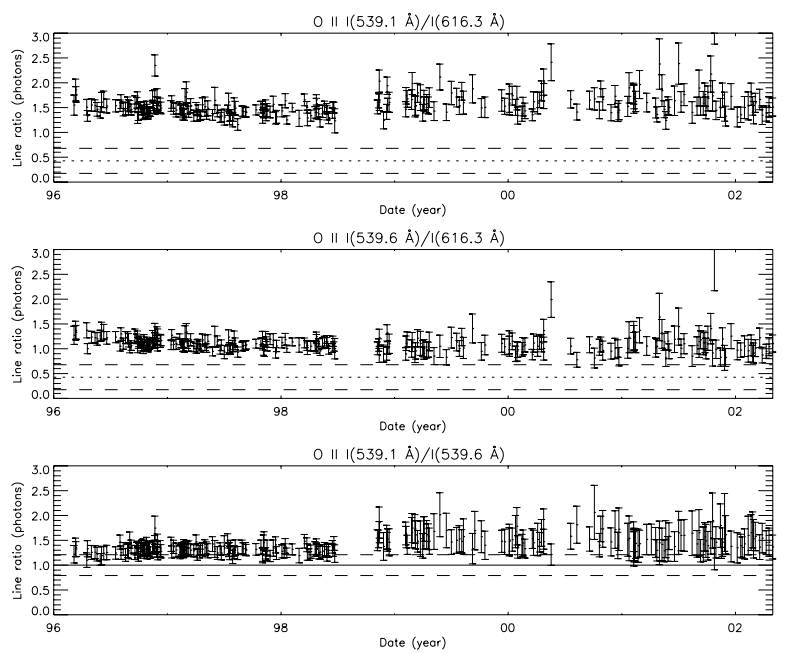

Fig. 3. Line intensity ratios for O II. For the ratio $I(539.1 \AA) / I(539.6 \AA)$, the solid line parallel to the abscissa is the theoretical line ratio. The other two ratios are density sensitive and the dotted line represents the theory at an electron temperature of $3.2 \times 10^{4} \mathrm{~K}$ and an electron density of $1 \times 10^{8} \mathrm{~cm}^{-3}$. The pairs of dashed lines in each of the three ratios represent the upper and lower ranges of the theory as determined by the addition in quadrature of the uncertainties in the theoretical line ratio and for each line the radiometric calibration and the burn-in correction. For the ratio $I(539.1 \AA) / I(539.6 \AA)$ the uncertainty in the radiometric calibration is taken as zero as the lines are very close in wavelength. The uncertainties in the measured ratios are obtained from the uncertainties as determined by the fitting routine.

the ratio during the first year of operations. However, the ratio remains the same after the attitude-loss of the spacecraft, albeit with a larger scatter. This indicates that the calibration has been maintained over the six years of observations.

\subsection{OIV}

Ratios involving members of the O IV $2 \mathrm{~s}^{2} 2 \mathrm{p}{ }^{2} \mathrm{P}-2 \mathrm{~s} 2 \mathrm{p}^{2}{ }^{2} \mathrm{P}$ multiplet at $554 \AA$ and the $2 \mathrm{~s}^{2} 2 \mathrm{p}{ }^{2} \mathrm{P}_{1 / 2}-2 \mathrm{~s} 2 \mathrm{p}^{2}{ }^{2} \mathrm{~S}_{1 / 2}$ transition at $608.4 \AA$ were used by both Landi et al. (1997) and Del Zanna et al. (2001). However, line ratios such as $I(554.5 \AA) / I(608.4 \AA)$, where the $554.5 \AA$ line is the strongest component $(3 / 2-3 / 2)$ of the ${ }^{2} \mathrm{P}-{ }^{2} \mathrm{P}$ multiplet, are sensitive to electron temperature. At $1.6 \times 10^{5} \mathrm{~K}$, the temperature of maximum ion population in ionisation equilibrium for O IV as given by Arnaud \& Rothenflug (1985), this line ratio is 5.9. It is 6.4 and 4.7 at $4.0 \times 10^{5} \mathrm{~K}$ and $6.0 \times 10^{4} \mathrm{~K}$ respectively, the temperatures at which the ion populations have reached zero. The atomic model used for the calculation employed A values from Froese Fischer (1983), Dankwort \& Treffetz (1978) and Merkelis et al. (1995) and the effective collision strengths of Zhang et al. (1994). The measured line intensity ratio $I(554.5 \AA) / I(608.4 \AA)$ is shown in Fig. 4 .

The weighted average of the data taken before the loss-ofattitude of SOHO is $7 \%$ higher than the ratio for the temperature of maximum ionisation population in ionisation equilibrium and agrees with theory well within the uncertainties. The present results also agree with those of Lanzafame et al. (2005). The data taken after the loss-of-attitude have lower uncertainties, their weighted average is $17 \%$ lower than theory and they also agree with theory within the uncertainties. However, the two sets of data do not overlap. Active region ratios show the same behaviour. Noting that the more intense member of the ${ }^{2} \mathrm{P}-{ }^{2} \mathrm{~S}$ doublet is blended with the strong $\mathrm{MgX} 609.79 \AA$ line 


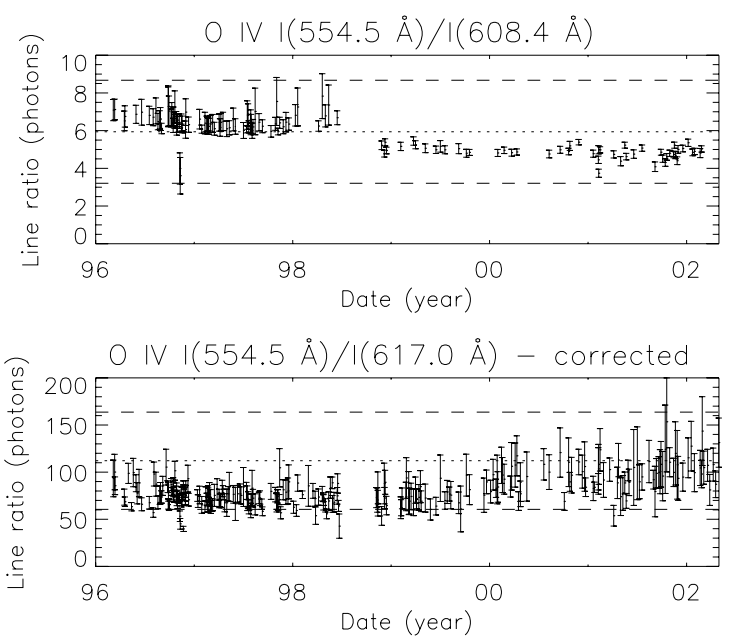

Fig. 4. The OIV line intensity ratios $I(554.5 \AA) / I(608.4 \AA)$ and $I(554.5 \AA) / I(617.0 \AA)$ corrected for the O II blend for the quiet Sun. In each graph the dotted line parallel to the abscissa is the theoretical line ratio at an electron temperature of $1.6 \times 10^{5} \mathrm{~K}$ while the dashed lines represent the upper and lower ranges as determined by the addition in quadrature of the uncertainties in the theoretical line ratio and for each line the burn-in correction and the radiometric calibration. The uncertainties in the measured ratios are obtained from the uncertainties as determined by the fitting routine.

(Brooks et al. 1999), the line intensity ratio $I(554.5 \AA) /$ $I(617.0 \AA)$ was investigated. This ratio is more temperature dependent than the $I(554.5 \AA) / I(608.4 \AA)$ ratio. The $617 \AA$ line is a blend of the members of the O IV $2 \mathrm{~s} 2 \mathrm{p}^{2}{ }^{2} \mathrm{D}-2 \mathrm{p}^{3}{ }^{2} \mathrm{P}$ multiplet and the O II $2 s^{2} 2 \mathrm{p}^{3}{ }^{2} \mathrm{D}_{3 / 2}-2 \mathrm{~s}^{2} 2 \mathrm{p}^{2} 3 \mathrm{~s}^{2} \mathrm{P}_{1 / 2}$ line. This blend can be corrected using the intensity of the other two members of the O II multiplet at $616.3 \AA$ and theory as just described for O II. The resulting ratio is given in Fig. 4.

The correction for the blend increases the uncertainty in the measured ratio. Note that the correction factor was as expected for the upper levels of the O II multiplet being in statistical equilibrium and thus the uncertainty in the factor was ignored. The ratio agrees with theory within the uncertainties. The step present in the previous ratio at the break in observations caused by the loss-of-attitude of SOHO is not present. Thus, it can be concluded there is a problem with the $608.4 \AA$ line after the loss-of-attitude. This line is in the wings of a very strong line and the problem is probably the burn-in correction compounded by the non-Gaussian shape of the post-recovery lines. The spectra moved on the detector as a result of the loss-of-attitude of SOHO and the movement put this line in the place where the strong Mg X $609.79 \AA$ line caused detector burn-in before the loss-of-attitude. The post-recovery changes in both the location of the spectral lines on the detector, and in the widths of the much stronger lines around it, combine to make the burn-in of the $608.4 \AA$ line one of the most difficult to determine in the whole NIS-2 spectrum. However, as the measured ratios still agree with theory within the uncertainty, it is not thought prudent to change the radiometric calibration at $608.4 \AA$ as the problem is almost certainly the burn-in correction.

\section{4. $\mathrm{Cax}$}

The Cax $3 \mathrm{~s}{ }^{2} \mathrm{~S}-3 \mathrm{p}{ }^{2} \mathrm{P}$ doublet at $557.76 \AA$ and $574.01 \AA$ is observed in NIS-2 spectra. Landi et al. (1997) report the latter line as blended in active region spectra and Del Zanna et al. (2001) find that it is blended in on-disc observations. Our quiet Sun measurements before the loss-of-attitude of SOHO agree with those of Lanzafame et al. (2005). The measurements of the ratio $I(557.8 \AA) / I(574.0 \AA)$ lie mostly below the lower uncertainties for the ratio, consistent with the $574.0 \AA$ line being blended. Using the average of the pre-loss measurements and the theory ratio, it is estimated that the blending line is about $40 \%$ of the intensity of the $574.01 \AA$ line itself. For about a year after the recovery of SOHO, the ratio compares well with the pre-loss measurements but subsequently falls off in intensity. It is difficult to disentangle the effect of a change in the intensity of the impurity line and any possible change in the calibration. However, this ratio supports the interpretation of the O IV $I(554.5 \AA) / I(617.0 \AA)$ ratio that there is no change in the NIS-2 radiometric calibration around the loss-of-attitude of SOHO.

\section{Monitoring the calibration of NIS-2 in second order}

In NIS-2 the Si XI $2 \mathrm{~s} 2 \mathrm{p}{ }^{1} \mathrm{P}-2 \mathrm{p}^{2}{ }^{1} \mathrm{D}$ line at $604.1 \AA$ is observed in first order close to the $2 \mathrm{~s}^{2}{ }^{1} \mathrm{~S}-2 \mathrm{~s} 2 \mathrm{p}{ }^{1} \mathrm{P}$ resonance line at 303.4 $\AA$ which is seen in second order in the wings of the second order He II 303.8 $\AA$ line. The 604.1 $\AA$ line is difficult to observe with CDS in the quiet Sun (Brooks et al. 1999; Lang et al. 2001; Lanzafame et al. 2005). Active region observations of the line were used by Landi et al. (1997) in their calibration study. Lang et al. (2001) also studied this ion. The atomic data which determine the population structure of the ion were reappraised. Calculations of line intensity ratios were done for a set of temperatures centered on $1.6 \times 10^{6} \mathrm{~K}$ which is the temperature of maximum ion population in the ionisation equilibrium calculations of Arnaud \& Rothenflug (1985). The line ratio $I(604.1 \AA) / I(303.4 \AA)$ is insensitive to electron density and temperature for solar conditions, the ratio depending on the ratio of electron excitation rates from the ground $2 \mathrm{~s}^{2}{ }^{1} \mathrm{~S}$ level to the $2 \mathrm{p}^{2}{ }^{1} \mathrm{D}$ and $2 \mathrm{~s} 2 \mathrm{p}{ }^{1} \mathrm{P}$ levels and the ratio of the $\mathrm{A}$ values for the transitions. With increasing density stepwise excitation to the $2 \mathrm{p}^{2}{ }^{1} \mathrm{D}$ level from the $2 \mathrm{~s} 2 \mathrm{p}{ }^{1} \mathrm{P}$ level increases and results in a density sensitivity of the ratio above a density of around $5 \times 10^{13} \mathrm{~cm}^{-3}$. A laboratory measurement of the line intensity ratio was made by König et al. (1996) using a theta-pinch discharge at a density of $3 \times 10^{16} \mathrm{~cm}^{-3}$ where the excitation of the $2 \mathrm{p}^{2}{ }^{1} \mathrm{D}$ level is predominately from the $2 \mathrm{~s} 2 \mathrm{p}{ }^{1} \mathrm{P}$ level. This measurement confirms the theoretical calculations within the experimental uncertainties. Following the uncertainties for the atomic data as estimated by Lang et al. (2001), namely $10 \%$ for theoretical intensities for lines excited from the ground level, the theoretical line ratio for solar conditions can be assigned an uncertainty of $14 \%$.

The line intensity ratio $I(604.1 \AA) / I(303.4 \AA)$ is shown in Fig. 5. The measured ratios agree with theory within the uncertainties. Most of the data points lie below the theoretical ratio. The loss-of-attitude of the spacecraft made no discernable change to the ratio, i.e., to the relative calibration of first and second order in NIS-2 at these wavelengths. There is some variation with time in the data taken well after the loss-of-attitude of SOHO. This may be a burn-in effect, as the $303.4 \AA$ line moved to where the red wing of the strong second order He II $303.8 \AA$ line was prior to the loss-of-attitude.

Other Si XI lines could also be used in conjunction with the 303.4 A line to cross calibrate NIS- 2 in second order. However, such ratios have greater theoretical uncertainty than the one adopted and were not utilized. 


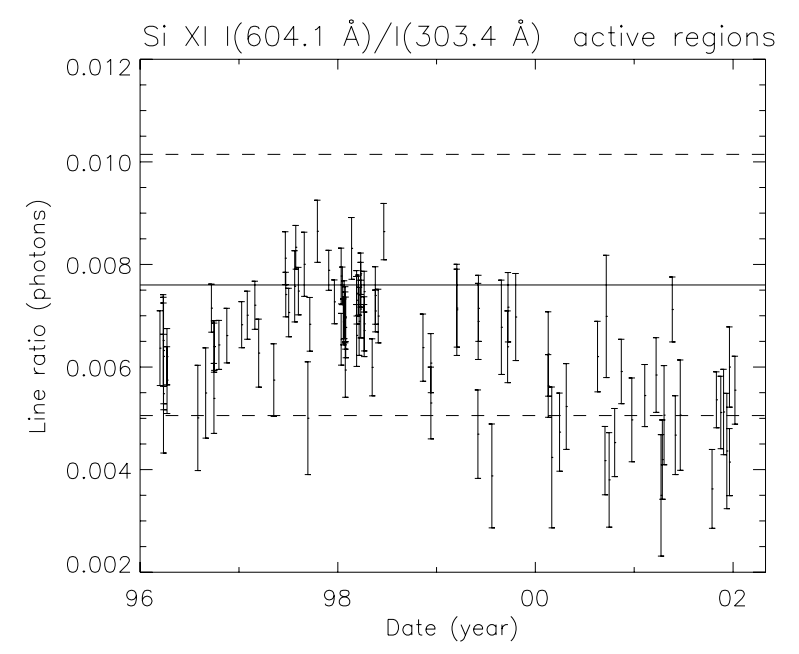

Fig. 5. The Si XI line intensity ratio $I(604.1 \AA) / I(303.4 \AA)$. The solid line parallel to the abscissa is the theoretical line ratio while the dashed lines represent the upper and lower ranges as determined by the addition in quadrature of the uncertainties in the theoretical line ratio and for each line the burn-in correction and the radiometric calibration. The uncertainties in the measured ratios are obtained from the uncertainties as determined by the fitting routine.

There is disagreement in the published identifications of second order NIS-2 lines. Brooks et al. (1999) only identified the Si XI $303.4 \AA$ and the He II $303.8 \AA$ lines. However, Del Zanna et al. (2001) in addition identified lines in second order which were given as unidentified by Brooks et al. (1999). Del Zanna et al. (2001) state that the Brooks et al. (1999) identifications "were based mostly on line position arguments" while their own "DEM analysis of each spectrum has enabled an accurate estimate of blending in each of the lines". They do not give details of their wavelength calibration so it is difficult to compare their wavelengths with those of Brooks et al. (1999). It should also be noted that Brooks et al. (1999) had four criteria for accepting the identification of a line, namely, adequate wavelength co-incidence, all members of a multiplet should be present at the expected relative separations and relative intensities, corresponding lines of isoelectronic sequences should appear at their appropriate wavelengths and intensities and the identifications should be consistent with the appearance of the position patterns. A position pattern for a line is a plot of the intensity of the line divided by the mean intensity of the line over all positions plotted logarithmically as a function of position index. The position index is an index used to identify each dataset and thus does not refer to the line position, rather it is an index of solar location. Therefore, the identifications were based not only on "line position arguments", but also on the consistent appearance of proposed lines in several datasets at different locations on the Sun and the consistency of their intensity variation between locations with that of other lines formed at the same temperature, as well as the other three criteria. These criteria should still be met, even with the use of a DEM. Some checks were carried out to see if the identifications proposed by Del Zanna et al. (2001) could be validated. The Mg VIII $2 \mathrm{~s}^{2} 2 \mathrm{p}{ }^{2} \mathrm{P}-2 \mathrm{~s} 2 \mathrm{p}^{2}{ }^{2} \mathrm{P}$ multiplet has four components, all seen in NIS-1 in first order. Three of these lie in the NIS-2 second order wavelength range. In second order, the 3/2-3/2 component was rejected by Brooks et al. (1999) as its wavelength co-incidence was inadequate and by Del Zanna et al. (2001) as it was masked by the strong O V line at $629.7 \AA$. Del Zanna (1999), the basis for the Del Zanna et al. (2001) publication, gives the component intensity ratio $I(1 / 2-1 / 2) / I(1 / 2-3 / 2)$ as $2.0(1.8)$ and $0.89(0.92)$ in NIS-1 and NIS-2 second order respectively, where the figures in parenthesis are those from Brooks et al. (1999). The ratio of the lines should be the same, not a factor of two different. This was one of the reasons the identification of these lines in second order was rejected by Brooks et al. (1999). The Si IX $2 \mathrm{~s}^{2} 2 \mathrm{p}^{2}{ }^{3} \mathrm{P}-2 \mathrm{~s} 2 \mathrm{p}^{3}{ }^{3} \mathrm{P}$ multiplet lies in one of the CDS GIS wavebands (GIS-2, $256 \AA$ to $338 \AA$ ) and in NIS-2 second order. Again there is a problem with the line ratios as observed in GIS-2 and NIS-2 second order which casts doubt on the Si IX identifications as given by Del Zanna (1999) and used in Del Zanna et al. (2001). It is difficult to check the use of single lines by Del Zanna et al. (2001) but note that the majority had been rejected by Brooks et al. (1999) mostly on wavelength and or position pattern considerations. Landi et al. (1997) also identify NIS-2 lines in second order in active region observations but do not give details of their wavelength calibration. For the present work, a cautious approach is adopted and the identifications by Del Zanna et al. (2001) and Landi et al. (1997) which cannot be checked are rejected. Thus we are left only with the Si XI ratio.

\section{Monitoring the intercalibration of NIS-2 and NIS-1}

\subsection{SiXI}

The Si XI $2 \mathrm{~s} 2 \mathrm{p}{ }^{3} \mathrm{P}-2 \mathrm{p}^{2}{ }^{3} \mathrm{P}$ multiplet lies in the NIS-1 wavelength range. Using CDS Lang et al. (2001) found that the multiplet could only be identified in active region spectra. Indeed, of the six lines of the multiplet, only the $2-1$ component at $371.5 \AA$ was unblended. The $2 \mathrm{~s}^{2}{ }^{1} \mathrm{~S}-2 \mathrm{~s} 2 \mathrm{p}{ }^{3} \mathrm{P}_{1}$ intercombination line is observed in the NIS- 2 spectrum at $580.92 \AA$. At low, i.e., quiet Sun densities, the $2 \mathrm{p}^{2}{ }^{3} \mathrm{P}$ term is excited both from the ground $2 \mathrm{~s}^{2}{ }^{1} \mathrm{~S}$ and $2 \mathrm{~s} 2 \mathrm{p}{ }^{3} \mathrm{P}$ levels giving density sensitivity to the $I(371.5 \AA) / I(580.9 \AA)$ ratio. The density dependence ceases when the excitation to the $2 \mathrm{p}^{2}{ }^{3} \mathrm{P}$ term is dominated by excitation from the metastable $2 \mathrm{~s} 2 \mathrm{p}{ }^{3} \mathrm{P}$ levels, i.e., just below $1 \times$ $10^{10} \mathrm{~cm}^{-3}$ to about $5 \times 10^{13} \mathrm{~cm}^{-3}$. Finally, when the $2 \mathrm{~s} 2 \mathrm{p}^{3} \mathrm{P}$ levels are depopulated by collisions rather than radiative decay, the line ratio again becomes density sensitive. In the present analysis, as for the previous Si XI line ratio, the atomic model and its uncertainties as given by Lang et al. (2001) are adopted. For this ratio, the laboratory measurement using a theta-pinch at a density $3 \times 10^{16} \mathrm{~cm}^{-3}$ is lower than theory. The same is true for the ratio of the $2 \mathrm{~s} 2 \mathrm{p}{ }^{3} \mathrm{P}_{2}-2 \mathrm{p}^{2}{ }^{3} \mathrm{P}_{1}$ line to the $2 \mathrm{~s}^{2}{ }^{1} \mathrm{~S}-2 \mathrm{~s} 2 \mathrm{p}{ }^{1} \mathrm{P}$ resonance line. In this case Lang et al. (2001) point out, following comparisons with laboratory measurements on a tokamak and theta-pinch discharges for lower-charged isoelectronic ions, that there could be a problem with the atomic model for triplet lines at theta-pinch densities. Brooks \& Warren (2006) suggest that the $580.92 \AA \mathrm{Si}$ XI line could be affected by blending with the $3 / 2-3 / 2$ and $1 / 2-3 / 2$ members of the O II $2 \mathrm{~s}^{2} 2 \mathrm{p}^{3}{ }^{2} \mathrm{P}-2 \mathrm{~s} 2 \mathrm{p}^{4}{ }^{2} \mathrm{P}$ multiplet at $580.97 \AA$. For the data taken before the loss-ofattitude the measured intensities of the O II blend at $616.3 \AA$ were used to estimate the intensities of the suggested blending lines and the Si XI line ratio corrected. The maximum correction was $1.3 \%$ and the average correction $0.06 \%$, consistent with the Si XI $580.92 \AA$ Aine being unblended with O II. Figure 6 shows the uncorrected line ratio for active region measurements where it is independent of density. The measurements lie below the theory, consistent with the $580.92 \AA$ line being blended, with some ratios agreeing within the uncertainties. There is no trend over 


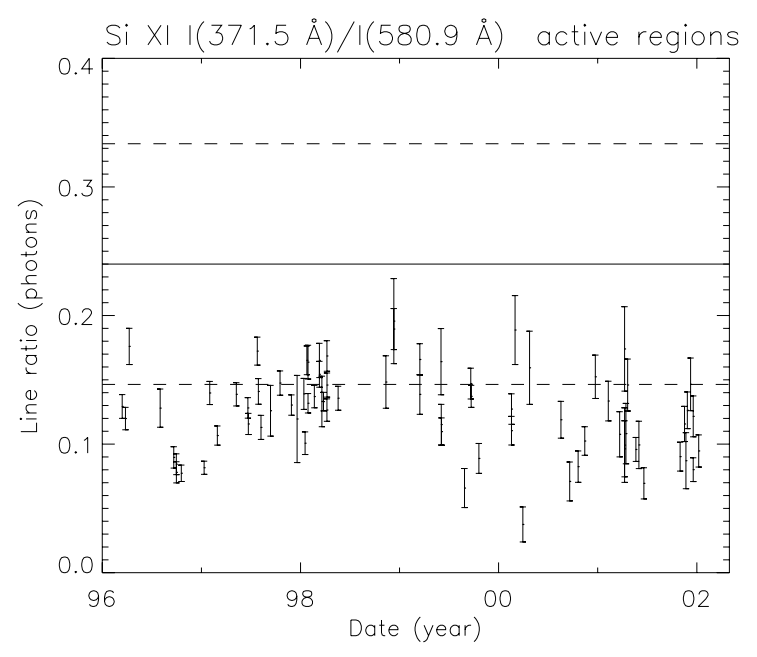

Fig. 6. The Si XI line intensity ratio $I(371.5 \AA) / I(580.9 \AA)$. The solid line parallel to the abscissa is the theoretical line ratio while the dashed lines represent the upper and lower ranges as determined by the addition in quadrature of the uncertainties in the theoretical line ratio and for each line the burn-in correction and the radiometric calibration. The uncertainties in the measured ratios are obtained from the uncertainties as determined by the fitting routine.

the six year period and no apparent change before and after the period of loss-of-attitude of the spacecraft.

Obviously other Si XI lines in NIS-2 could be used with the NIS-1 $375.1 \AA$ Si XI line, but in such cases the theoretical line ratio uncertainties were higher so the ratios were not used in the present work.

\section{2. $\mathrm{O} I I I$}

The O III $2 \mathrm{~s}^{2} 2 \mathrm{p}^{2}{ }^{3} \mathrm{P}-2 \mathrm{~s}^{2} 2 \mathrm{p} 3 \mathrm{~s}^{3} \mathrm{P}$ multiplet was identified in the quiet Sun spectrum by Brooks et al. (1999). The 1-2 component was found at $373.804 \AA$, a blend of the 0-1, 2-2 and 1-1 components at $374.097 \AA$, and a blend of the $1-0$ and $2-1$ components at $374.433 \AA$. The three ratios formed by pairs of these lines are shown in Fig. 7. The weighted means of the ratios are all higher than theory. As the ratios with the 1-2 component or the sum of the 1-0 and 2-1 components in the numerator are much higher compared to theory than the ratio between the two lines, i.e., $I(1-2) / I(1-0,2-1)$, it could be argued that these lines have unidentified blends. In their studies of the DEM of the quiet Sun using CDS Lanzafame et al. (2005) found ratios consistent with the present ratios. However, their DEM reconstruction of the line intensities was lower than the observations. They ruled out errors in the CDS NIS-1 to NIS-2 cross calibration as a cause of the discrepancy.

The atomic model used in ADAS to derive the line ratios used effective collision strengths and A values from Aggarwal \& Keenan (1999) and Weise et al. (1996). As discussed in Sect. 6, O III lines are identified in the NIS-2 spectra. The line intensity ratio $I(374.1 \AA) / I(599.6 \AA)$ is given in Fig. 8 as a function of electron temperature. The ratio is plotted for three electron densities and increases slightly with density. The temperature of maximum ion population in ionisation equilibrium for $\mathrm{O}$ III as given by Arnaud \& Rothenflug (1985) is $1.0 \times 10^{5} \mathrm{~K}$.

The line intensity ratio $I(374.1 \AA) / I(599.6 \AA)$ as measured using CDS is shown in Fig. 9. Below $3.2 \times 10^{4} \mathrm{~K}$ and above $3.2 \times 10^{5} \mathrm{~K}$ the $\mathrm{O}$ III ion populations in ionisation equilibrium as given by Arnaud \& Rothenflug (1985) are zero. Thus it is

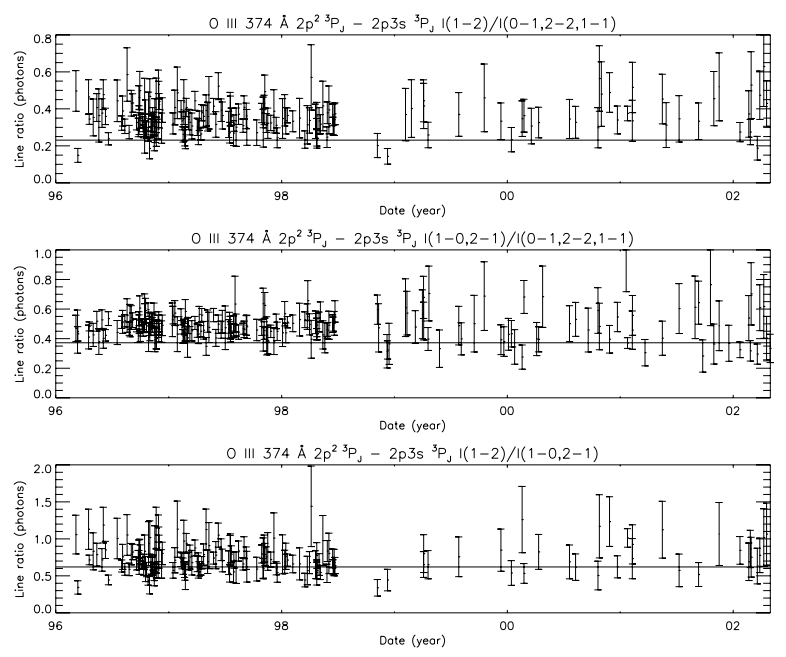

Fig. 7. Line intensity ratios between the observed components of the O III $2 s^{2} 2 p^{2}{ }^{3} \mathrm{P}-2 s^{2} 2 \mathrm{p} 3 \mathrm{~s}{ }^{3} \mathrm{P}$ multiplet. In each graph, the solid line parallel to the abscissa is the theoretical line ratio. The uncertainties in the measured ratios are obtained from the uncertainties as determined by the fitting routine.

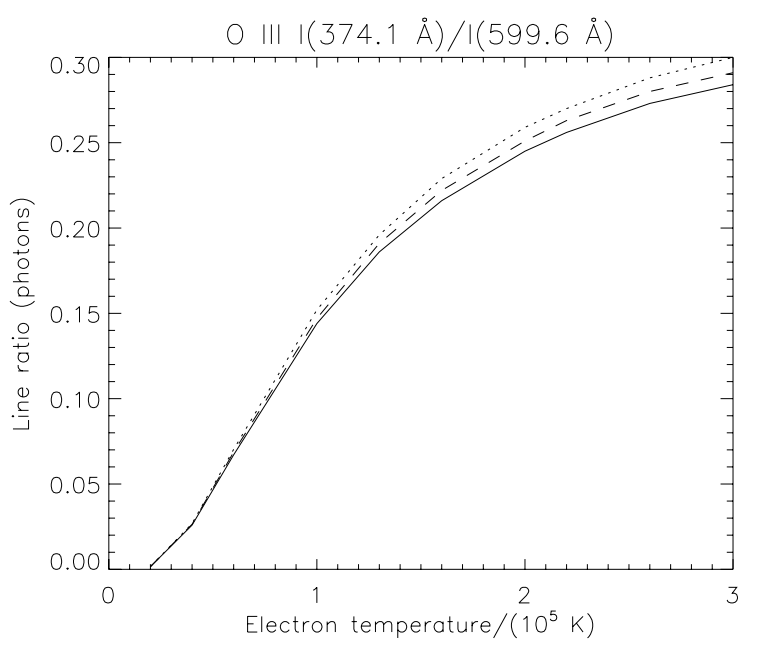

Fig. 8. The O III line intensity ratio $I(374.1 \AA) / I(599.6 \AA)$ as a function of electron temperature for three electron densities, $1 \times 10^{8} \mathrm{~cm}^{-3}$ (solid line), $1 \times 10^{9} \mathrm{~cm}^{-3}$ (dashed line) and $1 \times 10^{11} \mathrm{~cm}^{-3}$ (dotted line).

expected, as can be seen from Fig. 8, that the range of line intensity ratios is from about 0.014 to 0.3 . Inspection of Fig. 9 shows this to be the case within the uncertainties of the measured ratios, apart from one point. The majority of measured ratios lie within a much narrower range. Indeed the scatter of the measured ratios is not too much different from ratios where there is no dependence on plasma conditions and validates the use of this temperature dependent line ratio. As for the previous ratio (Si XI) there is no trend over the six year period and no apparent change before and after the period of loss-of-attitude of the spacecraft.

\section{3. $\mathrm{NeV}$}

Members of the NeV $2 s^{2} 2 p^{2}{ }^{3} \mathrm{P}-2 \mathrm{~s} 2 \mathrm{p}^{3}{ }^{3} \mathrm{D}$ multiplet were identified in the NIS-2 quiet Sun spectrum by Brooks et al. (1999), the 2-3 and 2-2 components being found blended at $572.311 \AA$. They also identified members of the $\mathrm{NeV}$ $2 s^{2} 2 p^{2}{ }^{3} \mathrm{P}-2 \mathrm{~s} 2 \mathrm{p}^{3}{ }^{3} \mathrm{~S}$ multiplet with the $2-1$ component being found at $359.374 \AA$ in the quiet Sun NIS-1 spectrum. The line 


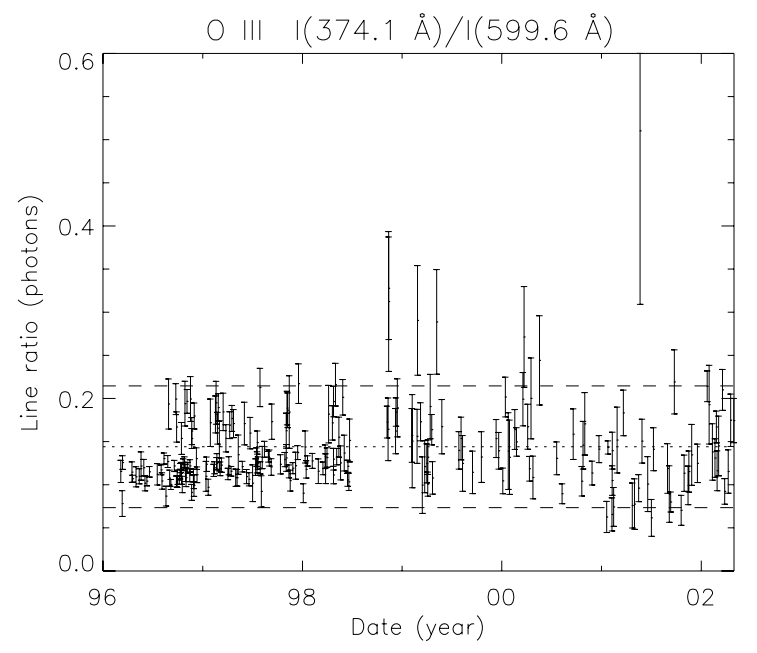

Fig. 9. The O III line intensity ratio $I(374.1 \AA) / I(599.6 \AA)$. The dotted line parallel to the abscissa is the theoretical line ratio at the temperature of maximum ion population in ionisation equilibrium for $\mathrm{O}$ III as given by Arnaud \& Rothenflug (1985). The dashed lines represent the upper and lower ranges as determined by the addition in quadrature of the uncertainties in the theoretical line ratio and for each line the burnin correction and the radiometric calibration. The uncertainties in the measured ratios are obtained from the uncertainties as determined by the fitting routine.

intensity ratio $I(359.374 \AA) / I(572.311 \AA)$ is given in Fig. 10 . The theoretical ratio was calculated using ADAS with the effective collision strengths and A values from the work of Griffin \& Badnell (2000). The ratio is sensitive to temperature but insensitive to density in the range from $1 \times 10^{8} \mathrm{~cm}^{-3}$ to $1 \times 10^{15} \mathrm{~cm}^{-3}$ and then increases with density. The temperature of maximum ion population in ionisation equilibrium for $\mathrm{Ne} \mathrm{V}$ as given by Arnaud \& Rothenflug (1985) is $3.2 \times 10^{5} \mathrm{~K}$. The experimental point is from the laboratory measurements by Lang (1988) where the spectral lines were produced using a theta-pinch discharge whose electron density was $6.2 \times 10^{15} \mathrm{~cm}^{-3}$ at the time of peak $\mathrm{Ne} \mathrm{V}$ emission. The theoretical ratio is $50 \%$ higher than the measurement. If the uncertainty in the theoretical intensity ratio is taken as $20 \%$, arising mainly from assigning an uncertainty of $15 \%$ to the effective collision strengths, experiment and theory agree within their uncertainties.

The ratio measured using CDS is shown in Fig. 11. Below $1 \times 10^{5} \mathrm{~K}$ and above $8 \times 10^{5} \mathrm{~K}$ the $\mathrm{Ne} \mathrm{V}$ ion populations in ionisation equilibrium as given by Arnaud \& Rothenflug (1985) are zero. Thus it is expected, as can be seen from Fig. 10, that the range of line intensity ratios is from about 0.12 to 0.50 . For the period before the loss-of-attitude of SOHO most of the measured line ratios lie above the value expected from theory for the temperature of maximum ion population in ionisation balance and some ratios lie above the theoretical ratio at the temperature at which the ion population has fallen to zero. The observations used by Lanzafame et al. (2005) in their DEM study were taken before the loss-of-attitude of SOHO. They found that the intensity of the $572 \AA$ blend was overestimated by their reconstruction. However, their DEM at the temperature of $\mathrm{Ne} \mathrm{V}$ was constrained by the other line in the present ratio. They ascribed the atomic data as a possible cause of the discrepancy as their study ruled out a possible inaccuracy in the NIS-1 to NIS-2 cross-calibration. The results taken before the loss-of-attitude indicate the usefulness of this temperature sensitive line ratio for calibration purposes. After the loss-of-attitude the results are

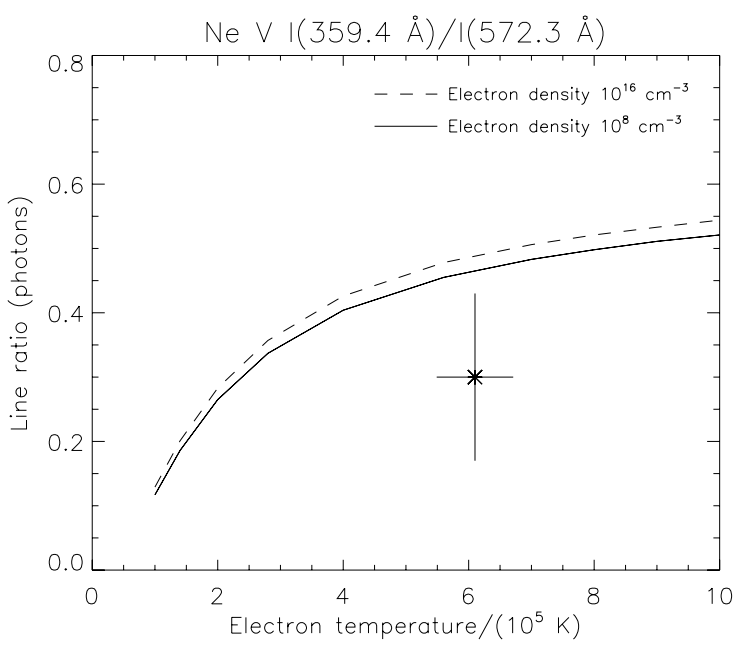

Fig. 10. The $\mathrm{NeV}$ line intensity ratio $I(359 \AA) / I(572 \AA)$, i.e., $I\left(2 \mathrm{~s}^{2} 2 \mathrm{p}^{2}{ }^{3} \mathrm{P}_{2}-2 \mathrm{~s} 2 \mathrm{p}^{3}{ }^{3} \mathrm{~S}_{1}\right) / I\left(2 \mathrm{~s}^{2} 2 \mathrm{p}^{2}{ }^{3} \mathrm{P}_{2}-2 \mathrm{~s} 2 \mathrm{p}^{3}{ }^{3} \mathrm{D}_{2,3}\right.$, as a function of electron temperature. The laboratory measurement of Lang (1988) obtained at an electron density of $6.2 \times 10^{15} \mathrm{~cm}^{-3}$ is indicated by the cross with error bars.

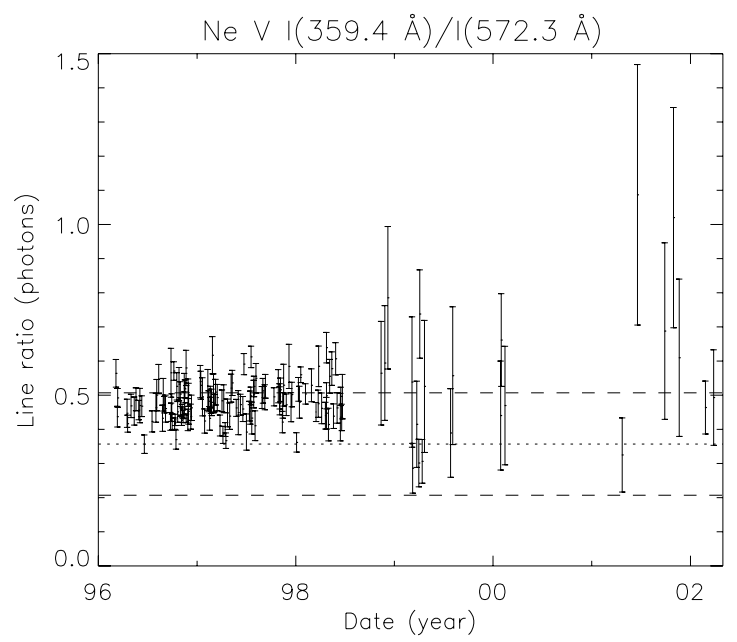

Fig. 11. The $\mathrm{Ne} V$ line intensity ratio $I(359 \AA) / I(572 \AA)$. The dotted line parallel to the abscissa is the theoretical line ratio at the temperature of maximum ion population in ionisation equilibrium for $\mathrm{Ne} \mathrm{V}$ as given by Arnaud \& Rothenflug (1985). The dashed lines represent the upper and lower ranges as determined by the addition in quadrature of the uncertainties in the theoretical line ratio and for each line the burn-in correction and the radiometric calibration. The uncertainties in the measured ratios are obtained from the uncertainties as determined by the fitting routine.

more sparse and varying. This is a direct result of the loss of resolution of NIS-1 after the loss-of-attitude. The Fe XIII lines identified at $359.777 \AA$ by Brooks et al. (1999) are difficult to resolve from the $\mathrm{Ne} \mathrm{V}$ line. However, the results indicate that there is no obvious change in the ratio after the loss-of-attitude.

\section{Monitoring the calibration of NIS-1}

\subsection{FeXI}

Brooks et al. (1999) identified all components of the Fe XI $3 \mathrm{~s}^{2} 3 \mathrm{p}^{4}{ }^{3} \mathrm{P}-3 \mathrm{~s} 3 \mathrm{p}^{5}{ }^{3} \mathrm{P}$ multiplet with the $2-2$ and $1-2$ components at $352.7 \AA$ and $369.2 \AA$, respectively, being unblended. This was confirmed by the DEM analysis of Lanzafame et al. (2005). 


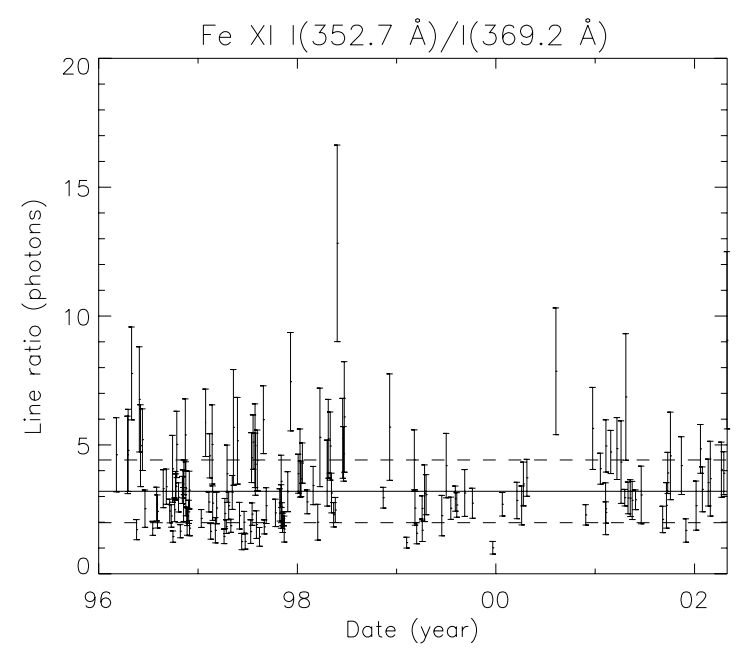

Fig. 12. The Fe XI line intensity ratio $I(352.7 \AA) / I(369.2 \AA)$. The solid line parallel to the abscissa is the theoretical branching ratio. The dashed lines represent the upper and lower ranges as determined by the addition in quadrature of the uncertainties in the theoretical line ratio and for each line the burn-in correction and the radiometric calibration. The uncertainties in the measured ratios are obtained from the uncertainties as determined by the fitting routine.

These lines form a branching ratio. In their critically evaluated data, Shirai et al. (2000) gave the A value ratio $\mathrm{A}(2-2) / \mathrm{A}(1-2)$ as $\left(1.7 \times 10^{9}\right) /\left(5.3 \times 10^{8}\right)=3.21$. In papers not included in the evaluation, Bhatia \& Doschek (1996) gave the ratio as $\left(2.529 \times 10^{9}\right) /\left(8.007 \times 10^{8}\right)=3.16$ and Fritzsche et al. $(2000)$ gave $\left(2.23 \times 10^{9}\right) /\left(6.98 \times 10^{8}\right)=3.20$. Although the ratio is 3.2 there are differences between the A values from the critically evaluated and the two later publications. Shirai et al. (2000) give the uncertainty in each of the A values as $\mathrm{C}$ where $\mathrm{C}$ stands for uncertainties within $25 \%$ and B for uncertainties within $10 \%$. For the branching ratio an uncertainty of $20 \%$ is adopted, noting that as a ratio is formed, some of the uncertainties in each A value will tend to cancel, as shown.

The ratio measured using CDS quiet Sun observations is shown in Fig. 12. There is no discernable change in the ratio after the loss-of-attitude or with time over the six years of operations. The weighted means of the ratio are $2.23 \pm 0.09$ and $2.41 \pm 0.14$ for observations before and after the loss-of-attitude, respectively. However, a large range of values has been measured for this branching ratio, even larger than the spread obtained in ratios dependent on the plasma conditions. The ratio has been measured using SERTS. Thomas \& Neupert (1994) obtained $3.28 \pm 0.58$ for active region observations from SERTS-89. Brosius et al. (1996) obtained $2.46 \pm 0.78,1.53 \pm 0.43$, and $4.00 \pm 1.88$ for quiet Sun, active region and off-limb observations, respectively, from SERTS-91. They also obtained $3.48 \pm$ 0.87 and $2.90 \pm 0.72$ for quiet Sun and active region observations from SERTS-93. As for the present observations, there is a large range of values, some of which do not agree within their uncertainties. This could be the result of occasional blending of the lines. If the theoretical ratio is correct, one line would have to be blended to give the low results while the other would need to be blended to give the high results.

On the whole the results validate the calibration within the uncertainties and do not provide any evidence that the calibration needs to be changed.

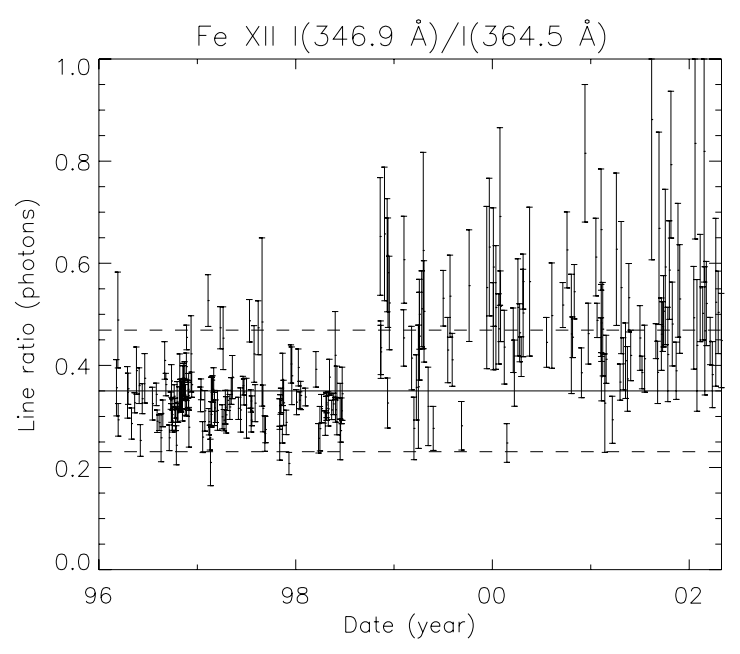

Fig. 13. The Fe XII $I(346.9 \AA) / I(364.5 \AA)$ quiet Sun line intensity ratio. The solid line parallel to the abscissa is the theoretical ratio. The dashed lines represent the upper and lower ranges as determined by the addition in quadrature of the uncertainties in the theoretical line ratio and for each line the burn-in and the radiometric calibration. The uncertainties in the measured ratios are obtained from the uncertainties as determined by the fitting routine.

\subsection{FeXII}

The intensity ratio $I(346.9 \AA) / I(364.5 \AA)$ of the $3 / 2-1 / 2$ and $3 / 2-5 / 2$ components of the Fe XII $3 s^{2} 3 p^{3}{ }^{4} S-3 s 3 p^{4}{ }^{4} \mathrm{P}$ multiplet is shown in Fig. 13 for quiet Sun observations. The line ratio (0.35), which is insensitive to electron density and temperature for quiet Sun and active regions, was evaluated using ADAS. The radiative data were from Binello et al. (2001) and the collision data from Tayal et al. (1987), for transitions within the ground configuration, and from Flower (1977), for transitions involving higher levels. Binello et al. (2001) obtain 0.31 using different collision strengths while Keenan et al. (1996) obtained 0.35 with slightly different atomic data, namely the data from Tayal \& Henry (1988) for transitions between the $3 \mathrm{~s}^{2} 3 \mathrm{p}^{3}$ and $3 \mathrm{~s} 3 \mathrm{p}^{4}$ levels. The estimated uncertainty in the ratio is $10 \%$, noting that changing atomic data makes little difference to the ratio, that as transitions within the same multiplet are being considered uncertainties will tend to cancel, and that the calculated ratio is close to that for the relative strength of multiplets $(0.33)$ as given by Allen (1976) (where it is assumed that the levels of the upper term in the multiplet are statistically populated).

The data taken before the loss-of-attitude are lower and less variable than the later data. The $346.9 \AA$ line is weak and difficult to measure. The post-loss situation with the change in line profile is worse than the pre-loss case, the post-loss fitting uncertainties being higher. In active region spectra the Si XI $2 s 2 \mathrm{p}^{3} \mathrm{P}-2 \mathrm{p}^{2}{ }^{3} \mathrm{P}$ multiplet is observed (e.g. Lang et al. 2001) and its 1-1 component at $364.494 \AA$ will blend with the $364.486 \AA$ Fe XII line. Young et al. (1998) estimate the contribution of the Si XI line to the blend in the SERTS-89 active region spectra of Thomas \& Neupert (1994) as 7\% while Landi et al. (1997) estimate the contribution in their active region spectrum to be less than $5 \%$. The Si XI line was not reported in the quiet Sun spectra of Brooks et al. (1999) and Del Zanna et al. (2001) noted the line was blended in active region but not in quiet Sun spectra. The results from the various SERTS observations again cover the range observed by CDS. For the SERTS-89 active region the ratio is $0.27 \pm 0.05$ (corrected to $0.30 \pm 0.05$ ) (Thomas \& Neupert 1994 and Young et al. 1998, respectively). The SERTS-91 
results are $0.56 \pm 0.11,0.53 \pm 0.11$ and $0.55 \pm 0.10$ for active region, quiet Sun and above limb spectra respectively and from SERTS-93 $0.43 \pm 0.07$ and $0.35 \pm 0.06$ for active region and quiet Sun (Brosius et al. 1996, uncorrected for Si XI). These results cover the range of values of the present quiet Sun CDS observations. This coupled with the fact that the mean of the postrecovery results is $36 \%$ higher than the pre-loss results but that many of the post-loss results still agree with theory make it difficult to conclude that this ratio indicates a calibration change because of the loss-of-attitude.

\section{3. $M g$ VIII}

All the lines of the Mg VIII $2 \mathrm{~s}^{2} 2 \mathrm{p}{ }^{2} \mathrm{P}-2 \mathrm{~s} 2 \mathrm{p}^{2}{ }^{2} \mathrm{P}$ multiplet are observed in NIS-1 both in quiet Sun and active region spectra.

The necessary theoretical ratios were obtained using ADAS. The A values needed were obtained from Merkelis et al. (1995) apart from that between the $1 / 2-3 / 2$ levels of the ground term which was taken from Froese Fischer (1983). The A values are estimated each to have uncertainties of $10 \%$ based on the work of Weise et al. (1996). The effective collision strengths needed for the calculations were from Zhang et al. (1994) and are estimated to have uncertainties of $15 \%$. The temperature of maximum ion population in ionisation equilibrium for $\mathrm{Mg}$ VIII as given by Arnaud \& Rothenflug (1985) is $7.9 \times 10^{5} \mathrm{~K}$.

The quiet Sun DEM analysis by Lanzafame et al. (2005) for data taken before the loss-of-attitude found that the $315.0 \AA$ line may be affected by a blend which contributes $50 \%$ of the line intensity, i.e., the fitted intensity of the line must be reduced by a factor 1.5. Although the measured branching ratio involving this line agreed with theory within the observational uncertainties, allowing for the blend gave much better agreement and also the electron densities measured using the non-branching line ratios agreed well. Applying this factor to quiet Sun observations taken before the loss-of-attitude of the spacecraft, the ratios in Fig. 14 are obtained. At densities above those of the quiet Sun, the non-branching line ratios become insensitive to density, reaching upper limits which are indicated in the figure.

The $I(313.7 \AA) / I(317.0 \AA)$ branching ratio is in good agreement with theory for observations before and after the period of the loss-of-attitude of the spacecraft. For the $I(315.0 \AA) / I(311.8 \AA)$ branching ratio, the data are sparse as there are difficulties in fitting the $311.8 \AA$ line in quiet Sun post-recovery data. For the density sensitive ratio $I(315.0 \AA) / I(317.0 \AA)$ the data are lower than the limit value as expected, since quiet Sun densities are below the high density limit and the ratio increases with increasing density, while the ratios taken before and after the spacecraft loss-of-attitude are in agreement, with larger scatter in the post-loss observations. Once more, a density sensitive line intensity ratio has been used to validate the calibration. For the $I(311.8 \AA) / I(317.0 \AA)$ ratio again difficulties in the fitting of the $311.8 \AA$ line preclude comparison with the earlier data.

Figure 15 shows active region data for the same ratios as in Fig. 14 with the same correction factor for the intensity of the $315.0 \AA$ line taken before the loss-of-attitude. The $I(313.7 \AA) / I(317.0 \AA)$ branching ratio is again in good agreement with theory. For the $I(315.0 \AA) / I(311.8 \AA)$ branching ratio, there are fewer points pre-recovery but more later and although the data are sparse, it can be concluded that the ratio is the same for the full period of observations. The $I(315.0 \AA) / I(317.0 \AA)$

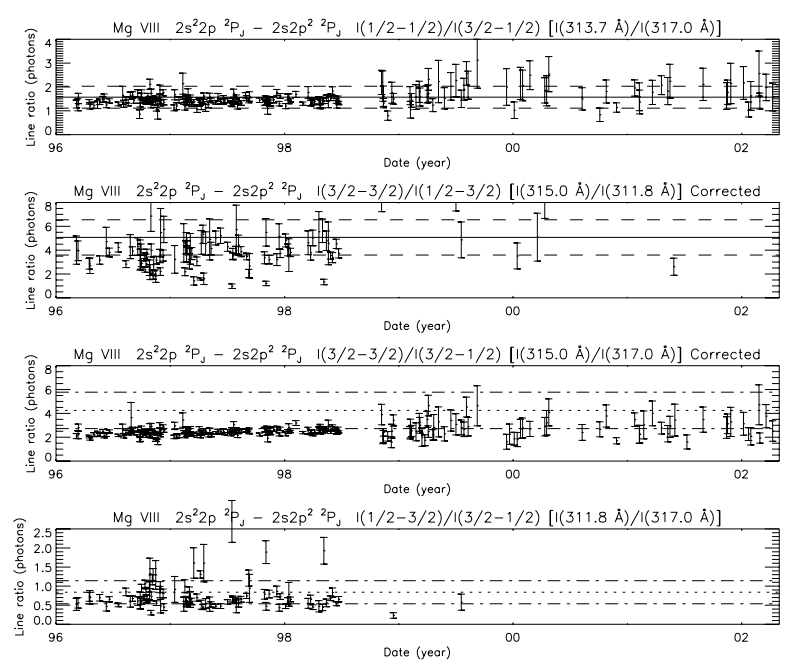

Fig. 14. Quiet Sun line intensity ratio for the Mg VIII $2 s^{2} 2 p{ }^{2} \mathrm{P}-2 \mathrm{~s} 2 \mathrm{p}^{2}{ }^{2} \mathrm{P}$ multiplet where the fitted intensities of the $315.0 \AA$ Aine in observations taken before the loss-of-attitude have been reduced by a factor 1.5 . In the two upper graphs, the solid lines parallel to the abscissa are the theoretical branching ratios. The dashed lines represent the upper and lower ranges as determined by the addition in quadrature of the uncertainties in the theoretical line ratio and for each line the burn-in correction and the radiometric calibration. In the two lower graphs, the dotted lines give the high density limit for the ratios, i.e., for densities greater than in the quiet Sun, and the dash dot lines represent the upper and lower ranges. The uncertainties in the measured ratios are obtained from the uncertainties as determined by the fitting routine.

ratio is lower than theory but still within the uncertainties and again the ratio is the same for the whole period of observation.

The correction factor to the measured $315.0 \AA$ line was applied to both quiet Sun and active region spectra for data taken before the loss-of-attitude. The presence of an unknown blend, as suggested by Lanzafame et al. (2005), who dealt only with quiet Sun data taken before the loss-of-attitude, is invalidated by the fact that the correction factor (1.5) is needed also for active region ratios. They suggested the blend, from a line formed at a temperature below that of Mg VIII formation, to give agreement with a similar study of the SERTS-89 active region spectrum (Lanzafame et al. 2002) which indicated no blending. Moreover, it should be emphasized that the correction was not made to data acquired post-recovery. A possible explanation of the data is that pre-recovery the responsivity locally at $315 \AA$ is wrong and that after recovery when the spectra shifted a few pixels on the detector, the line moved away from the problem area. The responsivity of NIS-1 in this wavelength region (see Lang et al. 2002) is determined from the analysis of coordinated measurement with the SERTS-97 rocket flight (Thomas 2002). The CDS responsivity is taken as a smooth curve through the spread of the SERTS-97 determined responsivity points. One of the comparison lines is in fact the $315.0 \AA$ line. The present results indicate that the CDS responsivity at $315 \AA$ should be increased by a factor 1.5 (as the intensity and responsivity are inversely proportional) and that the SERTS point at this wavelength should be increased slightly more than this factor as it lies below the CDS curve.

The active region results show that the $I(311.8 \AA) / I(317.0 \AA)$ ratio differs before and after the loss-of-attitude period, agreeing with theory before but being outside the combined uncertainties afterwards. The other ratio involving the $311.8 \AA$ lines has 


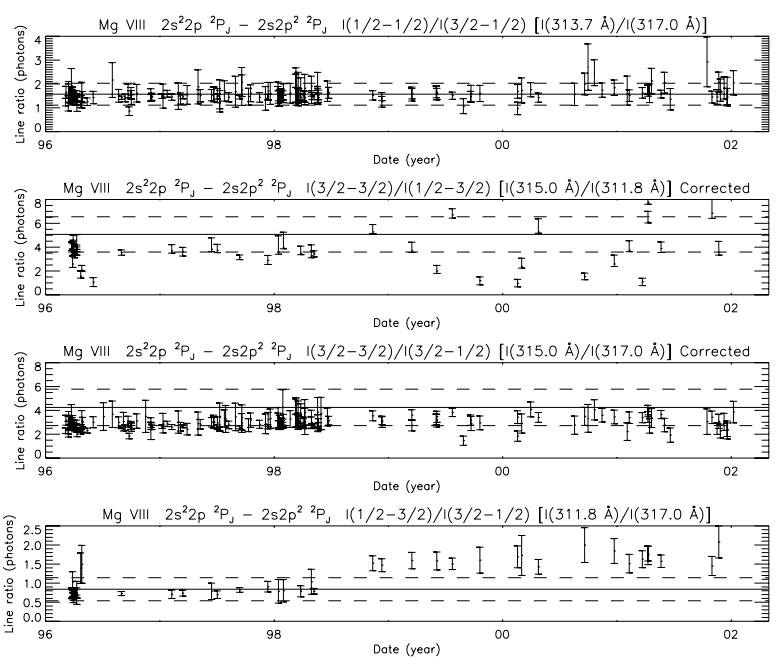

Fig. 15. Active region line intensity ratio for the $\mathrm{Mg}$ VIII $2 s^{2} 2 p{ }^{2} \mathrm{P}-2 \mathrm{~s} 2 \mathrm{p}^{2}{ }^{2} \mathrm{P}$ multiplet where the fitted intensities of the 315.0 A line in observations taken before the loss-of-attitude have been reduced by a factor 1.5. In each graph the solid line parallel to the abscissa is the theoretical ratio. In the two lower graphs the theoretical ratio is the high density limit, i.e., the constant value expected at active region densities. The dashed lines represent the upper and lower ranges as determined by the addition in quadrature of the uncertainties in the theoretical line ratio and for each line the burn-in correction and the radiometric calibration. The uncertainties in the measured ratios are obtained from the uncertainties as determined by the fitting routine.

sparse and variable data and does not rule out a problem with the $311.8 \AA$ line while ratios involving the $317.0 \AA$ line show agreement with theory. The $311.8 \AA$ line is outside the wavelength range of the SERTS-97 to CDS comparisons and thus the CDS responsivity at wavelengths below the SERTS-97 comparison range should perhaps be assigned higher uncertainties. The data indicate that the post-recovery responsivity at $311.8 \AA$ should be increased to give agreement with theory and the pre-recovery results. Within the uncertainties, the results from three SERTS flights given by Bhatia and Thomas (1998) agree with those of Figs. 14 and 15.

The Mg VIII $2 \mathrm{~s}^{2} 2 \mathrm{p}^{2} \mathrm{P}-2 \mathrm{~s} 2 \mathrm{p}^{2}{ }^{2} \mathrm{~S}$ doublet is also observed, the $1 / 2-1 / 2$ and $3 / 2-1 / 2$ transitions being at $335.231 \AA$ and $338.983 \AA$ respectively. The former is often blended and cannot be resolved from the $335.396 \AA$ Fe XVI line in quiet Sun spectra and is dominated by it in active region spectra. However, the line ratio $I\left(2 \mathrm{~s}^{2} 2 \mathrm{p}{ }^{2} \mathrm{P}_{1 / 2}-2 \mathrm{~s} 2 \mathrm{p}^{2}{ }^{2} \mathrm{P}_{1 / 2}\right) / I\left(2 \mathrm{~s}^{2} 2 \mathrm{p}{ }^{2} \mathrm{P}_{3 / 2}-2 \mathrm{~s} 2 \mathrm{p}^{2}{ }^{2} \mathrm{~S}_{1 / 2}\right)$, i.e., $I(313.74 \AA) / I(338.98 \AA)$, is density independent in active region spectra and density sensitive in quiet Sun spectra. The active region line intensity ratio $I(313.74 \AA) / I(338.98 \AA)$ is shown in Fig. 16. The $338.98 \AA$ line is difficult to fit in the post-loss data and this is reflected in the increase in line ratio uncertainties post-loss.

The theoretical results are obtained from the same ADAS data as used for the previous Mg VIII ratios. The prerecovery data yield a steady ratio while the post-recovery data show larger variation and are higher, but most points agree with theory within the uncertainties. A similar plot for the quiet Sun data shows the same consistency pre-loss while post-loss data have many points agreeing with the pre-loss data but still with the large spread in ratio as in the active region results. The results from three SERTS flights given by Bhatia \& Thomas (1998) agree with the pre-loss ratios. Attributing the change in ratio to

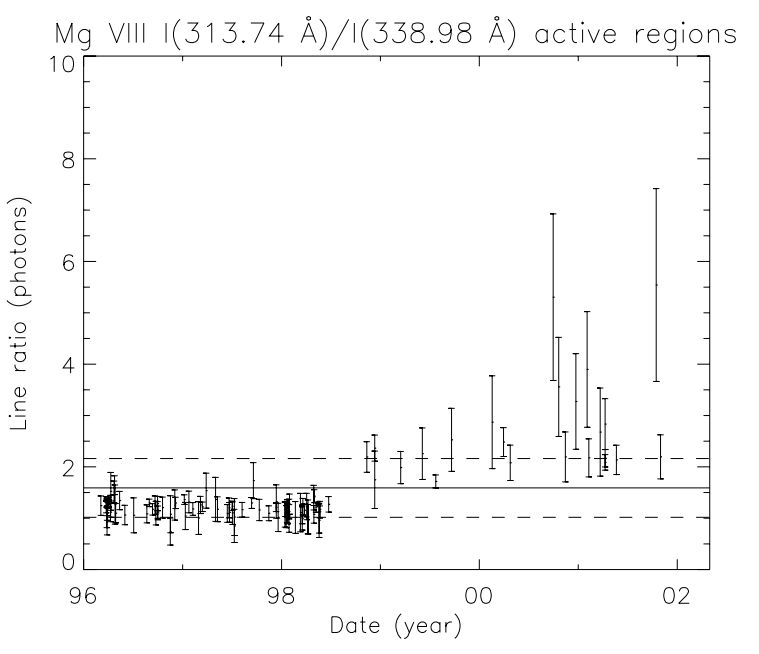

Fig. 16. The Mg VIII $I(313.74 \AA) / I(338.98 \AA)$ active region line intensity ratio. The solid line parallel to the abscissa is the theoretical ratio. The dashed lines represent the upper and lower ranges as determined by the addition in quadrature of the uncertainties in the theoretical line ratio and for each line the burn-in correction and the radiometric calibration. The uncertainties in the measured ratios are obtained from the uncertainties as determined by the fitting routine.

a change in the intensity of the $313.74 \AA$ line is inconsistent with the ratios just discussed. If the change were in the $338.98 \AA$ line, it has to be such that the line intensity is too low giving a high result for the ratio, which rules out blending in the less well-resolved post-recovery data but does not rule out changes in responsivity post-recovery. Although most of the post-recovery results in Fig. 16 agree with theory within the uncertainties, it appears that the post-recovery responsivity at $338.98 \AA$ needs to be changed because even though many points fall within the uncertainties they are now at the upper limit whereas pre-loss they were all near the lower limit.

\subsection{FeXVI}

The FeXVI $2 \mathrm{p}^{6} 3 \mathrm{~s}{ }^{2} \mathrm{~S}-2 \mathrm{p}^{6} 3 \mathrm{p}{ }^{2} \mathrm{P}$ doublet is observed at $335.396 \AA$ (1/2-3/2 component) and $360.743 \AA$ (1/2-1/2 component). The $335.396 \AA$ line is blended. The blend with the $1 / 2-1 / 2$ component (335.231 $\AA$ ) of the Mg VIII $2 s^{2} 2 p{ }^{2} \mathrm{P}-2 \mathrm{~s} 2 \mathrm{p}^{2}{ }^{2} \mathrm{~S}$ doublet is corrected using the intensity of the $3 / 2-1 / 2$ component at $338.98 \AA$ and the theory as given above for Mg VIII. The uncertainty in the correction is taken as $50 \%$. The Fe XII $3 \mathrm{~s}^{2} 3 \mathrm{p}^{3}{ }^{2} \mathrm{D}_{3 / 2}-3 \mathrm{~s} 3 \mathrm{p}^{4}{ }^{2} \mathrm{D}_{3 / 2}$ line at $335.06 \AA$ could also be present in the blend. This line was not present in the quiet Sun CDS spectrum of Brooks et al. (1999) but was observed in the SERTS-89 active region spectrum by Thomas \& Neupert (1994). However, in their DEM study of the SERTS spectrum Lanzafame et al. (2002) find that this line is too strong compared to observations by a factor of 2.6. Interestingly, Young et al. (1998) give the wavelength of the line as $335.34 \AA$ based on the work of Jupén et al. (1993) as opposed to the NIST Standard database (Martin et al. 1995). The only way to account for the blend of the Fe XII line with the Fe XVI line using CDS NIS observations is to use the intensity ratio of the Fe XII line to the Fe XII $3 s^{2} 3 p^{3}{ }^{2} D_{5 / 2}-3 s 3 p^{4}{ }^{2} D_{5 / 2}$ transition at $338.263 \AA$. Brosius et al. (1996), Young et al. (1998) and Lanzafame et al. (2002) all find this line is blended with an unknown line. Lanzafame et al. (2002) find that the 

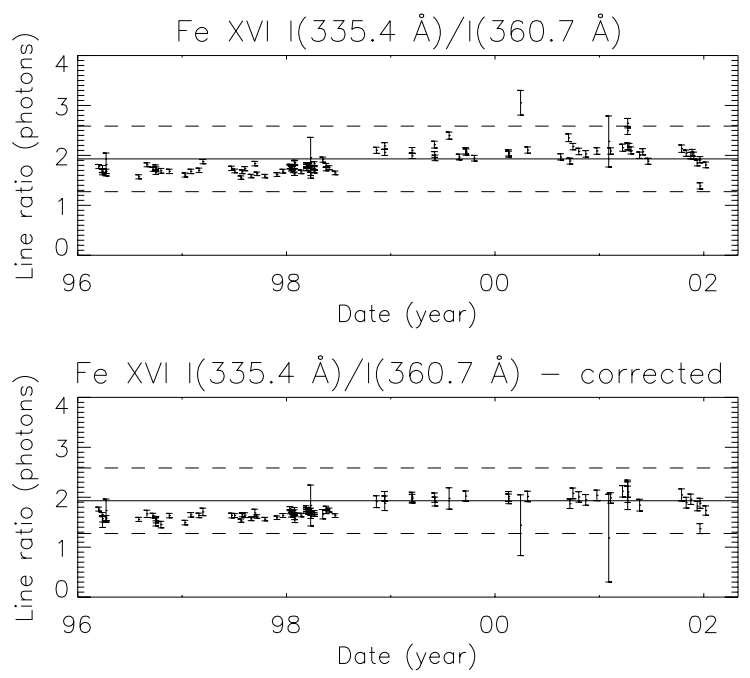

Fig. 17. The Fe XVI $I(335.4 \AA) / I(360.7 \AA)$ active region line intensity ratio. The solid lines parallel to the abscissa are the theoretical ratios. The dashed lines represent the upper and lower ranges as determined by the addition in quadrature of the uncertainties in the theoretical line ratio and for each line the burn-in correction and the radiometric calibration. The uncertainties in the measured ratios are obtained from the uncertainties as determined by the fitting routine.

measured intensity is $20 \%$ to $30 \%$ higher than theory. This is consistent with the results of Young et al. (1998) when it is noted that the line used in the branching ratio to obtain the estimate is marginally underestimated in the DEM of Lanzafame et al. (2002). Furthermore, the Fe XII ratio is density sensitive, being $4.1,2.4,1.2$, and 1.2 at densities of $1 \times 10^{8} \mathrm{~cm}^{-3}, 1 \times 10^{9} \mathrm{~cm}^{-3}$, $1 \times 10^{10} \mathrm{~cm}^{-3}$, and $1 \times 10^{11} \mathrm{~cm}^{-3}$ as evaluated using the atomic data discussed in Sect. 8.2. To obtain the correction, the blend is evaluated using the data of Lanzafame et al. (2002) and a factor of 1.2. The uncertainties in the blend and the line ratio corrections are each taken as $100 \%$.

The Fe XVI line intensity ratio $I(335.4 \AA) / I(360.7 \AA)$ is shown in Fig. 17. The average magnitude of the corrections for the Mg VIII and Fe XII blends are each $2 \%$ and are thus insensitive to the uncertainties assigned to the corrections. In the atomic model used to calculate the line ratio the atomic data needed were taken from Sampson et al. (1990). The uncertainty in the theoretical line ratio is taken to be $10 \%$, noting that it is close to the value expected for the relative strengths of multiplets as given by Allen (1976). The temperature of maximum ion population in ionisation equilibrium for Fe XVI as given by Arnaud \& Raymond (1994) is $2.5 \times 10^{6} \mathrm{~K}$ and the line ratio is insensitive to temperature and density for the temperatures over which the ion emits and active region densities. The agreement with theory for the ratio is within the uncertainties. There is a $17 \%$ increase in the weighted means of the ratios taken after the recovery of the spacecraft compared to the mean of the data taken before the loss-of-attitude. However, this cannot be directly related to changes in responsivity. As noted by Lang et al. (2002), the wide-slit burn-in is not fully known for these lines and could well account for the differences in the ratios before and after the loss of spacecraft attitude. The active region SERTS-89 results $(2.2 \pm 0.5$, Thomas \& Neupert 1994) and SERTS-91 and SERTS-93 results $(1.9 \pm 0.3$ and $2.0 \pm 0.3$, Brosius et al. 1996) agree with the present results.

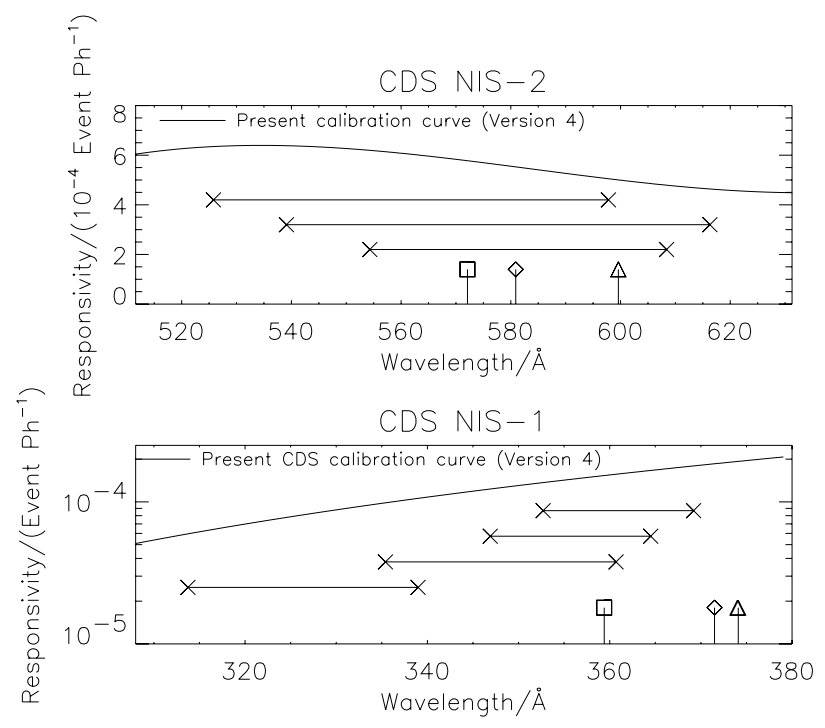

Fig. 18. For each of NIS-1, NIS-2 and NIS-2 second order, the wavelengths covered by the present analysis are indicated. Within NIS-1 and NIS-2 horizontal bars with crosses indicate the wavelength range of a line ratio. Vertical bars indicate a wavelength that is used to compare between the wavebands, the symbol above the bar indicating which pairs form each line ratio.

\section{5. $M g I X$}

It was noted in Lang et al. (2002) that there were indications that NIS-1 lost responsivity after the attitude-loss of SOHO by about a factor 1.5 at $368 \AA$ (Mg IX). This was based on a preliminary analysis of data taken using the SYNOP observing sequence which is run daily to give synoptic scans of $4^{\prime}$ wide rasters along the the solar meridian. A more sophisticated analysis to study network contrast and EUV irradiance (Thompson 2006) shows that the indications were false and the factor 1.5 need not be applied.

\section{Discussion and conclusions}

For NIS-2 the analysis indicates that the O III $525.8 \AA$ line is blended in active regions and that there are changes in the intensities of the O II 539.1 A line and the O IV 608.4 $\AA$ line, the latter probably as a result of difficulties in the burn-in correction after the loss-of-attitude of SOHO.

The O II, O III and O IV lines in NIS-2 give good wavelength coverage as shown in Fig. 18 where the responsivities of NIS-1 and NIS-2 are shown along with the line pairs used in the present work. For data taken before the loss-of-attitude of SOHO all the ratios agree with theory within the uncertainties, validating the radiometric calibration. For data taken after the loss-of-attitude, there are small changes in the $\mathrm{O}$ II and $\mathrm{O}$ III ratios while the O IV $(I(554.5 \AA) / I(617 \AA))$ ratio increases slightly with time. These changes are within the uncertainties of the measurements. There is no reason to change the radiometric calibration.

For NIS-2 in second order the measurements of the Si XI $303.4 \AA$ line could be affected by a burn-in effect in the data taken after the loss-of-attitude. However, the data taken before loss-of-attitude validates the calibration and although the ratio falls in later years, there is no need to change the calibration. The ratio used calibrates the NIS- 2 in second order for both the 
Si XI and He II lines which are the only lines conclusively identified in this wavelength band.

For the NIS-2 and NIS-1 inter-comparison line ratios from $\mathrm{O}$ III, $\mathrm{Ne} \mathrm{V}$ and Si XI validate the calibration, albeit that the Si XI ratios are at the limit of the uncertainties with some outside the limit. This is in agreement with the work of Lanzafame et al. (2005). The ratios also indicate that the loss-of-attitude of SOHO did not cause any change in the intercalibration.

For NIS-2, the ratios from Fe XI and Fe XII are found to be variable as in other measurements. Both could be affected by unknown blends. The Mg VIII ratio involving the $2 s^{2} 2 p{ }^{2} \mathrm{P}-2 \mathrm{~s} 2 \mathrm{p}^{2}{ }^{2} \mathrm{~S}$ line agrees with other measurements for data taken before the loss-of-attitude and there could well be a problem with the radiometric calibration at $338.98 \AA$ although most of the post-recovery results agree with theory within the uncertainties. The other $\mathrm{Mg}$ VIII ratios indicate that there is a problem with the calibration at $315.0 \AA$ for data taken before the loss-of-attitude of SOHO and that the responsivity at $311.8 \AA$ should perhaps have higher uncertainties and that the radiometric calibration could be changed to give agreement with results taken before the loss-of-attitude. The Fe XVI ratio also agrees with theory. The measurements taken before the loss-ofattitude are lower and less variable than those taken afterwards. The burn-in is not fully known for these lines and could account for the differences. Apart from the measurements of the $338.98 \AA, 311.8 \AA$, and $315.0 \AA$ lines, the results indicate that the radiometric calibration of NIS- 2 is validated.

The line ratios measured in the present investigation indicate that overall, apart from three specific wavelengths, the relative radiometric calibration is validated, yielding results which agree with theory within the uncertainties. Moreover, the calibration is maintained over the years of observation. Problems with the burn-in correction account for difficulties at $608.4 \AA$, $303.4 \AA$ (seen in second order), $335.4 \AA$, and $360.7 \AA$.

It can be considered that the NIS-2, NIS-2 second order and NIS-1 to NIS-2 inter-comparisons relate the calibrations at the lower wavelength range of NIS-2, the second order NIS-2 wavelengths and the higher end of the NIS-1 wavelength range with the longer wavelength range of NIS-2, as shown in Fig. 18. In addition, the NIS-1 ratios relate the shorter wavelength in that channel to the longer wavelengths and thus to the longer wavelengths in NIS-2. The radiometric intercalibration of CDS and the SUMER experiment on SOHO was done for lines in the longer wavelength range of NIS-2 (584 $\AA, 609 \AA$, and $624 \AA$ ) covering the first 4.6 years of joint observations and indicates that the radiometric calibration in the long wavelength part of NIS-2 was maintained (Pauluhn et al. 2002b). The present results thus extend this result to the whole NIS range with the exception of three wavelengths.

The line ratios method has been extended successfully from the use of branching ratios and ratios which are independent of plasma conditions to the use of ratios which are density or temperature sensitive.

Acknowledgements. CDS was built and is operated by a consortium led by the CCLRC Rutherford Appleton Laboratory and including the Mullard Space Science Laboratory, the NASA Goddard Space Flight Center, the Max Planck Institute for Extraterrestrial Physics, Garching and Oslo University. SOHO is a mission of international cooperation between ESA and NASA. This work was done in the context of the ADAS project. The authors thank P. Young for useful discussions and C. Burghardt for his help in preparing the paper. The authors would also like to thank the anonymous referee for helping to improve the clarity of the paper.

\section{References}

Aggarwal, K. M., \& Keenan, F. P. 1999, A\&AS, 123, 311

Allen, C. W. 1976, Astrophysical Quantities (London: Athlone Press)

Arnaud, M., \& Rothenflug, R. 1985, A\&AS, 60, 245

Arnaud, M., \& Raymond, J. C. 1994, ApJ, 398, 394

Bell, K. L., Hibbert, A., Stafford, R. P., \& McLaughlin, B. M. 1994, Phys. Scr., 50,343

Bhatia, A. K., \& Doschek, G. 1996, At. Data and Nucl. Data Tables, 64, 183

Bhatia, A. K., \& Thomas, R. J. 1998, ApJ, 497, 483

Binello, A. M., Landi, E., Mason, H. E., Storey, P. J., \& Brosius, J. W. 2001, A\&A, 370, 1071

Brooks, D. H., \& Warren, H. P. 2006, ApJS, 164, 202

Brooks, D. H., Fischbacher, G. A., Fludra, A., et al. 1999, A\&A, 347, 277

Brosius, J. W., Davila, J. D., Thomas, R. J., \& Monsignori-Fossi, B. C. 1996, ApJS, 106, 143

Dankwort, E., \& Treffetz, E. 1978, A\&AS, 65, 93

Delaboudinière, J.-P., Artzner, G. E., Brunaud, L., et al. 1995, Sol. Phys., 162, 291

Del Zanna, G. 1999, Ph.D. Thesis, University of Central Lancashire, Preston

Del Zanna, G., Bromage, B. J. I., Landi, E., \& Landini, M. 2001, A\&A, 379, 708

Flower, D. R. 1977, A\&A, 54, 163

Fritzsche, S., Dong, C. Z., \& Träbert, E. 2000, MNRAS, 318, 263

Froese Fischer, C. 1983, J. Phys. B, 16, 157

Griffin, D. C., \& Badnell, N. R. 2000, J. Phys. B, 33, 4389

Griffin, W. G., \& McWhirter, R. W. P. 1962, in Conf. on Optical Instruments and

Techniques, ed. K. J. Habell (London: Chapman and Hall), 14

Harrison, R. A., Sawyer, E. C., Carter, M. K., et al. 1995, Sol. Phys., 162, 233

Jupén, C., Isler, R. C., \& Träbert, E. 1993, MNRAS, 264, 267

Keenan, F. P., Thomas, R. J., Neupert, W. M., Foster, V. J., \& Tayal, S. S. 1996, MNRAS, 278, 773

König, R., Kolk, K.-H., \& Kunze, H.-J. 1996, Phys. Scr., 53, 679

Landi, E., Landini, M., Pike, C. D., \& Mason, H. E. 1997, Sol. Phys., 175, 553

Landi, E., Storey, P. J., \& Zeippen, C. J. 2004, ApJ, 607, 640

Lang, J. 1988, J. de Phys., 49, C1-59

Lang, J., Mason, H. E., \& McWhirter, R. W. P. 1990, Sol. Phys., 129, 31

Lang, J., Kent, B. J., Breeveld, A. A., et al. 2000, J. Opt. A, 2, 88

Lang, J., Brooks, D. H., O’Mullane, M. G., et al. 2001, Sol. Phys., 201, 37

Lang, J., Thompson, W. T., Pike, C. D., Kent, B. J., \& Foley, C. R. 2002, in The Radiometric Calibration of SOHO, ISSI Scientific Report SR-002, ed. A. Pauluhn, M. C. E. Huber, \& R. von Steiger, 105

Lanzafame, A. C., Brooks, D. H., Lang, J., et al. 2002, A\&A, 384, 242

Lanzafame, A. C., Brooks, D. H., \& Lang, J. 2005, A\&A, 432, 1063

Martin, W. C., Sugar, J., Musgrove, A., \& Dalton, G. R. 1995, NIST Database for Atomic Spectroscopy, Version 1.0, NIST Standard Reference Database 61

McLaughlin, B. M., \& Bell, K. L. 1994, ApJS, 94, 825

McLaughlin, B. M., \& Bell, K. L. 1998, J. Phys. B, 31, 4317

Merkelis, G., Vilkas, M. J., Gaigalas, G., \& Kisielius, R. 1995, Phys. Scr., 51, 233

Neupert, W. M., \& Kastner, S. O. 1983, A\&A, 128, 181

Pauluhn, A., Huber, M. C. E., \& von Steiger, R. (eds) 2002a, The Radiometric Calibration of SOHO, ISSI Scientific Report SR-002

Pauluhn, A., Lang, J., Schühle, U., et al. 2002b, in The Radiometric Calibration of SOHO, ISSI Scientific Report SR-002, ed. A. Pauluhn, M. C. E. Huber, \& R. von Steiger, 235

Sampson, D. H., Zhang, H. L., \& Fontes, C. 1990, At. Data and Nucl. Data Tables, 44, 209

Shirai, T., Sugar, J., Musgrove, A., \& Weise, W. L. 2000, J. Phys. Chem. Ref. Data, Mono. 8

Summers, H. P. 1994, ADAS Manual, JET Joint Undertaking Report JET$\operatorname{IR}(94) 07$

Summers, H. P. 2001, ADAS Manual, version 2-3, http://adas.phys.strath.ac.uk

Tayal, S. S., \& Henry, R. J. W. 1988, ApJ, 329, 1023

Tayal, S. S., Henry, R. J. W., \& Pradhan, A. K. 1987, ApJ, 319, 951

Thomas, R. J. 2002, in The Radiometric Calibration of SOHO, ISSI Scientific Report SR-002, ed. A. Pauluhn, M. C. E. Huber, \& R. von Steiger, 225

Thomas, R. J., \& Neupert, W. M. 1994, ApJS, 91, 461

Thompson, W. T. 2006, in preparation

Thompson, W. T., Poland, A. I., Sigmund, O. H. W., et al. 1992, SPIE Proc., 1743, 464

Weise, W. L., Fuhr, J. R., \& Deters, T. M. 1996, J. Phys. Chem. Ref. Data, Mono., 7

Young, P. R., Landi, E., \& Thomas, R. J. 1998, A\&A, 329, 291

Zhang, H. L., Graziani, M., \& Pradhan, A. K. 1994, A\&A, 283, 319 